\title{
REPRESENTATIONS OF THE LIE ALGEBRA OF VECTOR FIELDS ON A TORUS AND THE CHIRAL DE RHAM COMPLEX
}

\author{
YULY BILLIG AND VYACHESLAV FUTORNY \\ Dedicated to Yuri Alexandrovich Bahturin
}

\begin{abstract}
The goal of this paper is to study the representation theory of a classical infinite-dimensional Lie algebra - the Lie algebra Vect $T^{N}$ of vector fields on an $N$-dimensional torus for $N>1$. The case $N=1$ gives a famous Virasoro algebra (or its centerless version - the Witt algebra). The algebra $\operatorname{Vect} \mathbb{T}^{N}$ has a natural class of tensor modules parametrized by finitedimensional modules of $g l_{N}$. Tensor modules can be used in turn to construct bounded irreducible modules for Vect $\mathbb{T}^{N+1}$ (induced from $\operatorname{Vect} \mathbb{T}^{N}$ ), which are the focus of our study. We solve two problems regarding these bounded modules: we construct their free field realizations and determine their characters. To solve these problems we analyze the structure of the irreducible $\Omega^{1}\left(\mathbb{T}^{N+1}\right) / d \Omega^{0}\left(\mathbb{T}^{N+1}\right) \rtimes \operatorname{Vect} \mathbb{T}^{N+1}$-modules constructed in a paper by the first author. These modules remain irreducible when restricted to the subalgebra Vect $\mathbb{T}^{N+1}$, unless they belong to the chiral de Rham complex, introduced by Malikov-Schechtman-Vaintrob (1999).
\end{abstract}

\section{INTRODUCTION}

In this paper we study the representation theory of a classical infinite-dimensional Lie algebra - the Lie algebra Vect $T^{N}$ of vector fields on a torus. This algebra has a class of representations of a geometric nature, tensor modules, since vector fields act on tensor fields of any given type via the Lie derivative. Tensor modules are parametrized by finite-dimensional representations of $g l_{N}$, with the fiber of a tensor bundle being a $g l_{N}$-module.

Irreducible $g l_{N}$-modules yield tensor modules that are irreducible over $\operatorname{Vect} \mathbb{T}^{N}$, with the exception of the modules of differential $k$-forms. In the latter case, the $g l_{N}$-module is irreducible, yet the modules of $k$-forms are reducible, which follows from the fact that the differential of the de Rham complex is a homomorphism of Vect $\mathbb{T}^{N}$-modules. In the present paper we give a vertex algebra analogue of this result.

In the case of a circle, a conjecture of Kac, proved by Mathieu [21], states that for the Lie algebra of vector fields on a circle an irreducible weight module with finite-dimensional weight spaces is either a tensor module or a highest/lowest weight module. There is a generalization of this conjecture to an arbitrary $N$ due to

Received by the editors August 31, 2011 and, in revised form, September 11, 2012.

2010 Mathematics Subject Classification. Primary 17B66, 17B67; Secondary 17B69.

The first author was supported in part by a grant from the Natural Sciences and Engineering Research Council of Canada.

The second author was supported in part by the CNPq grant (301743/2007-0) and by the Fapesp grant (2010/50347-9). 
Eswara Rao [7]; see 23] for the results towards this conjecture. The analogues of the highest weight modules in this case are defined using the technique introduced by Berman-Billig [1. These modules are bounded with respect to one of the variables. It follows from a general result of [1] that irreducible bounded modules for the Lie algebra of vector fields on a torus have finite-dimensional weight spaces; however, the method of [1 yields no information on the dimensions of the weight spaces. This is the question that we solve in the present paper - we find explicit realizations of the irreducible bounded modules. Using these realizations the dimensions of the weight spaces may be readily determined.

A partial solution of this problem for the 2-dimensional torus was given by BilligMolev-Zhang [3] using non-commutative differential equations in vertex algebras. The algebra of vector fields on $\mathbb{T}^{2}$ contains the loop algebra $\widetilde{s l}_{2}=\mathbb{C}\left[t_{0}, t_{0}^{-1}\right] \otimes s l_{2}$. This subalgebra plays an important role in the representation theory of Vect $\mathbb{T}^{2}$. According to the results of [3], some of the bounded modules for Vect $T^{2}$ remain irreducible when restricted to the subalgebra $\widetilde{s l}_{2}$. Futorny classified in [1] irreducible generalized Verma modules for $\widetilde{s l}_{2}$. Such generalized Verma modules admit the action of the much larger algebra Vect $T^{2}$.

This relation between representations of $\widetilde{s l}_{2}$ and Vect $\mathbb{T}^{2}$ suggests that for the Lie algebra of vector fields on $\mathbb{T}^{N}$, an important role is played by its subalgebra $\widetilde{s l}_{N}$. An unexpected twist here is that it is not the generalized Verma modules for $\widetilde{s l}_{N}$ that admit the action of Vect $\mathbb{T}^{N}$ for $N>2$, but rather the generalized Wakimoto modules. The generalized Wakimoto modules are $\widetilde{s l}_{N}$-modules that have the same character as the generalized Verma modules, but need not be isomorphic to them.

The generalized Wakimoto modules for $\widetilde{s l}_{N}$ that we use here were constructed in [2] in the context of the representation theory of toroidal Lie algebras. However, their special properties with respect to the loop subalgebra $\widetilde{s l}_{N}$ were not previously recognized.

Let us outline the result of [2] that we use here. Since one of the variables plays a special role, it will be more convenient to work with an $(N+1)$-dimensional torus. To construct a full toroidal algebra, one begins with the algebra of $\dot{\mathfrak{g}}$-valued functions on $\mathbb{T}^{N+1}$ :

$$
\operatorname{Map}\left(\mathbb{T}^{N+1}, \dot{\mathfrak{g}}\right) \cong \mathbb{C}\left[t_{0}^{ \pm 1}, t_{1}^{ \pm 1}, \ldots, t_{N}^{ \pm 1}\right] \otimes \dot{\mathfrak{g}}
$$

where $\dot{\mathfrak{g}}$ is a finite-dimensional simple Lie algebra. Next we take the universal central extension of this multiloop algebra, with the center realized as the quotient of 1 -forms on the torus by differentials of functions [14]:

$$
\mathcal{K}=\Omega^{1}\left(\mathbb{T}^{N+1}\right) / d \Omega^{0}\left(\mathbb{T}^{N+1}\right) .
$$

Finally, one adds the Lie algebra of vector fields on the torus:

$$
\left(\mathbb{C}\left[t_{0}^{ \pm 1}, \ldots, t_{N}^{ \pm 1}\right] \otimes \dot{\mathfrak{g}} \oplus \mathcal{K}\right) \rtimes \operatorname{Vect} \mathbb{T}^{N+1} .
$$

Irreducible bounded modules for this Lie algebra were constructed in [2] using vertex algebra methods. Note that the results of [2] admit a specialization to $\dot{\mathfrak{g}}=(0)$. The multiloop algebra then disappears, leaving behind, like the grin of the Cheshire Cat, the space of its central extension:

$$
\mathcal{K} \rtimes \operatorname{Vect} \mathbb{T}^{N+1} .
$$


It turns out that it is easier to study representations of this Lie algebra, rather than of vector fields alone, because of the duality between vector fields and 1forms. Representation theory of this larger Lie algebra is controlled by a tensor product of three vertex algebras:

$$
V_{H y p}^{+} \otimes V_{g l_{N}} \otimes V_{V i r}
$$

a subalgebra of a hyperbolic lattice vertex algebra, the affine $\widehat{g l}_{N}$ vertex algebra at level 1 and the Virasoro vertex algebra of rank 0 . The tensor product of the first two components, $V_{H y p}^{+} \otimes V_{g l_{N}}$ is one of the bounded modules for $\mathcal{K} \rtimes \operatorname{Vect}^{N+1}$, and in fact it is a generalized Wakimoto module for the subalgebra $\widetilde{s l}_{N+1} \subset \operatorname{Vect} \mathbb{T}^{N+1}$. Then the results of [3] suggest that there is a chance that the $\mathcal{K} \rtimes \operatorname{Vect} \mathbb{T}^{N+1}$-modules constructed in 2] remain irreducible when restricted to Vect $\mathbb{T}^{N+1}$. The study of this question is the main part of the present paper. The answer that we get is remarkably parallel to the classical picture with the tensor modules. We prove that a bounded irreducible $\mathcal{K} \rtimes$ Vect $\mathbb{T}^{N+1}$-module remains irreducible when restricted to the subalgebra of vector fields, unless it belongs to the chiral de Rham complex, introduced by Malikov-Schechtman-Vaintrob 20] (for arbitrary manifolds).

It is only in very special situations that an irreducible module remains irreducible when restricted to a subalgebra. A prime example of this is the basic module for an affine Kac-Moody algebra, which remains irreducible when restricted to the principal Heisenberg subalgebra. This exceptional property of the basic module leads to its vertex operator realization and is at the heart of several spectacular applications of this theory.

The space of the chiral de Rham complex is the vertex superalgebra

$$
V_{H y p}^{+} \otimes V_{\mathbb{Z}^{N}}
$$

where $V_{\mathbb{Z}^{N}}$ is the lattice vertex superalgebra associated with the standard euclidean lattice $\mathbb{Z}^{N}$. The vertex superalgebra $V_{\mathbb{Z}^{N}}$ is graded by fermionic degree,

$$
V_{\mathbb{Z}^{N}}=\bigoplus_{k \in \mathbb{Z}} V_{\mathbb{Z}^{N}}^{k}
$$

and the components

$$
V_{H y p}^{+} \otimes V_{\mathbb{Z}^{N}}^{k}
$$

are irreducible $\mathcal{K} \rtimes$ Vect $\mathbb{T}^{N+1}$-modules. Yet for these modules the restriction to the Lie algebra of vector fields is no longer irreducible since the differential of the chiral de Rham complex

$$
\mathbf{d}: V_{H y p}^{+} \otimes V_{\mathbb{Z}^{N}}^{k} \rightarrow V_{H y p}^{+} \otimes V_{\mathbb{Z}^{N}}^{k+1}
$$

is a homomorphism of Vect $\mathbb{T}^{N+1}$-modules.

In fact it was noted in [18, that these components admit the action of the Lie algebra $\mathbb{C}\left[t_{0}, t_{0}^{-1}\right] \otimes \operatorname{Vect}^{N}$, but here we prove a much stronger result.

In conclusion, we make two curious observations. The Lie algebra of vector fields on a torus has a trivial center, yet its representation theory is described in terms of vertex algebras $V_{H y p}^{+}$and $V_{g l_{N}}$ that involve non-trivial central extensions. The central charges of these tensor factors cancel out to give a vertex algebra of total rank 0 .

A final remark is that the chiral de Rham complex is an essentially super object, whereas the Lie algebra of vector fields that we started with is classical. 
The structure of the paper is as follows. We introduce the main objects of our study in Sections 2, 3 and 4. We discuss vertex algebras and their applications to the representation theory of Vect $\mathbb{T}^{N+1}$ in Sections 5 and 6 . In Section 7 we introduce the generalized Wakimoto modules for the loop algebra $\widetilde{s l}_{N+1}$. We construct a non-degenerate pairing for the bounded modules in Section 8. We prove the main result on irreducibility in Section 9 and make a connection with the chiral de Rham complex in the final section of the paper.

\section{LiE ALGEBRA OF VECTOR FIELDS AND ITS TENSOR MODULES}

We begin with the algebra of Fourier polynomials on an $N$-dimensional torus $\mathbb{T}^{N}$. Introducing the variables $t_{j}=e^{i x_{j}}, j=1, \ldots, N$, we realize the algebra of functions as Laurent polynomials $\mathbb{C}\left[t_{1}^{ \pm 1}, \ldots, t_{N}^{ \pm 1}\right]$. The Lie algebra of vector fields on a torus is

$$
\operatorname{Vect} \mathbb{T}^{N}=\operatorname{Der} \mathbb{C}\left[t_{1}^{ \pm 1}, \ldots, t_{N}^{ \pm 1}\right]=\bigoplus_{p=1}^{N} \mathbb{C}\left[t_{1}^{ \pm 1}, \ldots, t_{N}^{ \pm 1}\right] \frac{\partial}{\partial t_{p}} .
$$

It will be more convenient for us to work with the degree derivations $d_{p}=t_{p} \frac{\partial}{\partial t_{p}}$ as the free generators of Vect $\mathbb{T}^{N}$ as a $\mathbb{C}\left[t_{1}^{ \pm 1}, \ldots, t_{N}^{ \pm 1}\right]$-module:

$$
\operatorname{Vect}^{N}=\bigoplus_{p=1}^{N} \mathbb{C}\left[t_{1}^{ \pm 1}, \ldots, t_{N}^{ \pm 1}\right] d_{p}
$$

The Lie bracket in $\operatorname{Vect} \mathbb{T}^{N}$ is then written as

$$
\left[t^{r} d_{a}, t^{m} d_{b}\right]=m_{a} t^{r+m} d_{b}-r_{b} t^{r+m} d_{a}, \quad a, b=1, \ldots, N .
$$

Here we are using the multi-index notation $t^{r}=t_{1}^{r_{1}} \ldots t_{N}^{r_{N}}$ for $r=\left(r_{1}, \ldots, r_{N}\right) \in$ $\mathbb{Z}^{N}$.

The Cartan subalgebra $\left\langle d_{1}, \ldots, d_{N}\right\rangle$ acts on $\operatorname{Vect}^{N}$ diagonally and induces a $\mathbb{Z}^{N}$-grading on it.

The Lie algebra of vector fields (on any manifold) has a class of representations of a geometric nature. Vector fields act via the Lie derivative on the space of tensor fields of a given type. Locally, on the tangent space at a point, tensors admit the action of $g l_{N}$, where vectors form a natural module and covectors its dual. Higher order tensors are obtained by taking tensor products of the natural and the conatural modules. Translating from the geometric language into algebraic, it is possible to express the Lie derivative action using just the $g l_{N}$-module structure on the tensors. Let us present the construction of tensor modules in the case of a torus $\mathbb{T}^{N}$.

Fix a finite-dimensional $g l_{N}$-module $W$. In the case when $W$ is irreducible, the identity matrix acts as multiplication by a scalar $\alpha \in \mathbb{C}$. Let $\gamma \in \mathbb{C}^{N}$. We define the tensor module $T=T(W, \gamma)$ to be the vector space

$$
T=q^{\gamma} \mathbb{C}\left[q_{1}^{ \pm 1}, \ldots, q_{N}^{ \pm 1}\right] \otimes W
$$

with the action given by

$$
t^{r} d_{a}\left(q^{\mu} \otimes w\right)=\mu_{a} q^{\mu+r} \otimes w+\sum_{p=1}^{N} r_{p} q^{\mu+r} \otimes E^{p a} w,
$$


where $r \in \mathbb{Z}^{N}, \mu \in \gamma+\mathbb{Z}^{N}, a=1, \ldots, N$ and $E^{p a}$ is the matrix with 1 in the $(p, a)$-position and zeros elsewhere.

Theorem 2.1 ([6]; cf. [27]). Let $W$ be an irreducible finite-dimensional $\mathrm{gl}_{\mathrm{N}^{-}}$ module. The tensor module $T(W, \gamma)$ is an irreducible Vect $^{N}$-module, unless it appears in the de Rham complex of differential forms:

$$
q^{\gamma} \Omega^{0}\left(\mathbb{T}^{N}\right) \stackrel{\mathbf{d}}{\longrightarrow} q^{\gamma} \Omega^{1}\left(\mathbb{T}^{N}\right) \stackrel{\mathbf{d}}{\longrightarrow} \ldots \stackrel{\mathbf{d}}{\longrightarrow} q^{\gamma} \Omega^{N}\left(\mathbb{T}^{N}\right) .
$$

The middle terms in this complex are reducible $\mathrm{Vect}^{N}{ }^{N}$-modules, while the modules $q^{\gamma} \Omega^{0}\left(\mathbb{T}^{N}\right)$ and $q^{\gamma} \Omega^{N}\left(\mathbb{T}^{N}\right)$ are reducible whenever $\gamma \in \mathbb{Z}^{N}$.

Note that the de Rham differential $\mathbf{d}$ is a homomorphism of $\operatorname{Vect} \mathbb{T}^{N}$-modules. Let us specify the irreducible $g l_{N}$-modules that correspond to the tensor modules in the de Rham complex. The module of functions $\Omega^{0}$ and the module of differential $N$ forms $\Omega^{N}$ correspond to 1-dimensional $g l_{N}$-modules $W$ on which the identity matrix acts as multiplication by $\alpha=0$ and $\alpha=N$ respectively. The remaining modules $\Omega^{k}, k=1, \ldots, N-1$, are the highest weight modules for $s l_{N}$ with the fundamental highest weights $\omega_{k}$ and $\alpha=k$ (see e.g. [6]). Even though they correspond to irreducible $g l_{N}$-modules, tensor modules of differential forms are reducible since the kernels and images of the differential $\mathbf{d}$ are obviously submodules in $\Omega^{k}$.

\section{BOUNDED MODUles}

Our goal is to generalize to an arbitrary $N$ the category of the highest weight modules over Vect $\mathbb{T}^{N}$. In our constructions one of the coordinates will play a special role. From now on we will be working with the $N+1$-dimensional torus and will index our coordinates as $t_{0}, t_{1}, \ldots, t_{N}$, where $t_{0}$ is the "special variable". We would like to construct modules for the Lie algebra $\mathcal{D}=\operatorname{Vect} \mathbb{T}^{N+1}$ in which the "energy operator" $-d_{0}$ has spectrum bounded from below.

Let us consider a $\mathbb{Z}$-grading of $\mathcal{D}$ by degrees in $t_{0}$. This $\mathbb{Z}$-grading induces a decomposition

$$
\mathcal{D}=\mathcal{D}_{-} \oplus \mathcal{D}_{0} \oplus \mathcal{D}_{+}
$$

into subalgebras of positive, zero and negative degrees in $t_{0}$. The degree zero part is

$$
\mathcal{D}_{0}=\bigoplus_{p=0}^{N} \mathbb{C}\left[t_{1}^{ \pm 1}, \ldots, t_{N}^{ \pm 1}\right] d_{p}
$$

In particular, $\mathcal{D}_{0}$ is a semi-direct product of the Lie algebra of vector fields on $\mathbb{T}^{N}$ with the abelian ideal $\mathbb{C}\left[t_{1}^{ \pm 1}, \ldots, t_{N}^{ \pm 1}\right] d_{0}$.

We begin the construction of a bounded module by taking a tensor module for $\mathcal{D}_{0}$. Fix a finite-dimensional irreducible $g l_{N}$-module $W, \beta \in \mathbb{C}$ and $\gamma \in \mathbb{C}^{N}$. We define a $\mathcal{D}_{0}$-module $T$ as the space

$$
T=q^{\gamma} \mathbb{C}\left[q_{1}^{ \pm 1}, \ldots, q_{N}^{ \pm 1}\right] \otimes W
$$

with the tensor module action (2.1) of the subalgebra $\operatorname{VectT}^{N} \subset \mathcal{D}_{0}$ and with $\mathbb{C}\left[t_{1}^{ \pm 1}, \ldots, t_{N}^{ \pm 1}\right] d_{0}$ acting by shifts:

$$
t^{r} d_{0}\left(q^{\mu} \otimes w\right)=\beta q^{\mu+r} \otimes w .
$$

Next we let $\mathcal{D}_{+}$act on $T$ trivially and define $M(T)$ as the induced module

$$
M(T)=\operatorname{Ind}_{\mathcal{D}_{0} \oplus \mathcal{D}_{+}}^{\mathcal{D}} T \cong U\left(\mathcal{D}_{-}\right) \otimes T .
$$


The module $M(T)$ has a weight decomposition with respect to the Cartan subalgebra $\left\langle d_{0}, \ldots, d_{N}\right\rangle$ and (the real part of) the spectrum of $-d_{0}$ on $M(T)$ is bounded from below. However, the weight spaces of $M(T)$ that lie below $T$ are all infinitedimensional.

It turns out that the situation improves dramatically when we pass to the irreducible quotient of $M(T)$. One can immediately see that the Lie algebra $\mathcal{D}$ belongs to the class of Lie algebras with polynomial multiplication (as defined in [1]), whereas tensor modules belong to the class of modules with polynomial action. A general theorem of [1] (see also 4]) yields in this particular situation the following:

Theorem 3.1 ([1]). (i) The module $M(T)$ has a unique maximal submodule $M^{\text {rad }}$. (ii) The irreducible quotient $L(T)=M(T) / M^{\text {rad }}$ has finite-dimensional weight spaces.

This leads to the following natural problems:

Problem 1. Determine the character of $L(T)$.

Problem 2. Find a realization of $L(T)$.

In [3] these problems were solved for some of the modules $L(T)$ in the case of a 2 -dimensional torus $(N=1)$. In the present paper we will give a solution in full generality for any $N$.

\section{Toroidal Lie algebras}

For a finite-dimensional simple Lie algebra $\dot{\mathfrak{g}}$ we consider a multiloop algebra $\mathbb{C}\left[t_{0}^{ \pm 1}, \ldots, t_{N}^{ \pm 1}\right] \otimes \dot{\mathfrak{g}}$. Its universal central extension has a realization with center $\mathcal{K}$ identified as the quotient space of 1-forms by differentials of functions [14,

$$
\mathcal{K}=\Omega^{1}\left(\mathbb{T}^{N+1}\right) / d \Omega^{0}\left(\mathbb{T}^{N+1}\right) .
$$

The Lie bracket in

$$
\mathbb{C}\left[t_{0}^{ \pm 1}, \ldots, t_{N}^{ \pm 1}\right] \otimes \dot{\mathfrak{g}} \oplus \mathcal{K}
$$

is given by

$$
\left[f_{1}(t) \otimes g_{1}, f_{2}(t) \otimes g_{2}\right]=f_{1} f_{2} \otimes\left[g_{1}, g_{2}\right]+\left(g_{1} \mid g_{2}\right) \overline{f_{2} d f_{1}}
$$

where $g_{1}, g_{2} \in \dot{\mathfrak{g}}, f_{1}, f_{2} \in \mathbb{C}\left[t_{0}^{ \pm 1}, \ldots, t_{N}^{ \pm 1}\right],(\cdot \mid \cdot)$ is the Killing form on $\dot{\mathfrak{g}}$ and denotes the projection $\Omega^{1} \rightarrow \Omega^{1} / d \Omega^{0}$.

We will set 1 -forms $k_{a}=t_{a}^{-1} d t_{a}, a=0, \ldots, N$, as generators of $\Omega^{1}\left(\mathbb{T}^{N+1}\right)$ as a free $\mathbb{C}\left[t_{0}^{ \pm 1}, \ldots, t_{N}^{ \pm 1}\right]$-module. We will use the same notation for their images in $\mathcal{K}$.

The Lie algebra $\mathcal{D}=\operatorname{Vect} \mathbb{T}^{N+1}$ acts on the universal central extension of the multiloop algebra with the natural action on $\mathbb{C}\left[t_{0}^{ \pm 1}, \ldots, t_{N}^{ \pm 1}\right] \otimes \dot{\mathfrak{g}}$ and the action on $\mathcal{K}$ induced from the Lie derivative action of vector fields on $\Omega^{1}$ :

$$
f_{1}(t) d_{a}\left(f_{2}(t) k_{b}\right)=f_{1} d_{a}\left(f_{2}\right) k_{b}+\delta_{a b} f_{2} d\left(f_{1}\right), a, b=0, \ldots, N .
$$

The full toroidal Lie algebra is a semi-direct product

$$
\mathfrak{g}=\left(\mathbb{C}\left[t_{0}^{ \pm 1}, \ldots, t_{N}^{ \pm 1}\right] \otimes \dot{\mathfrak{g}} \oplus \mathcal{K}\right) \rtimes \mathcal{D} .
$$

In fact [2] treats a more general family of Lie algebras, where the Lie bracket in $\mathfrak{g}$ is twisted with a 2-cocycle $\tau \in H^{2}\left(\mathcal{D}, \Omega^{1} / d \Omega^{0}\right)$. However, for the purposes of the present work we need to consider only the semi-direct product, i.e., set $\tau=0$. 
A category of bounded modules for the full toroidal Lie algebra is studied in 2 and realizations of irreducible modules in this category are given. The constructions of [2] admit a specialization $\dot{\mathfrak{g}}=(0)$, which yields representations of the semi-direct product

$$
\mathcal{D} \ltimes \mathcal{K} .
$$

The approach of the present paper is to look at the representations of this semidirect product, constructed in [2], and to study their reductions to the subalgebra $\mathcal{D}$ of vector fields on $\mathbb{T}^{N+1}$. Surprisingly, as we shall see below, most of the irreducible modules for $\mathcal{D} \ltimes \mathcal{K}$ remain irreducible when restricted to $\mathcal{D}$.

In order to describe the results of [2] in this paper, we will need to present some background material on vertex algebras.

\section{Vertex superalgebras: Definitions and notation}

Let us recall the basic notions of the theory of vertex operator (super) algebras. Here we are following $[12$ and 17 .

Definition 5.1. A vertex superalgebra is a $\mathbb{Z}_{2}$-graded vector space $V$ with a distinguished vector $\mathbb{1}$ (vacuum vector) in $V$, a parity-preserving operator $D$ (infinitesimal translation) on the space $V$, and a linear map $Y$ (state-field correspondence)

$$
\begin{gathered}
Y(\cdot, z): \quad V \rightarrow(\operatorname{End} V)\left[\left[z, z^{-1}\right]\right], \\
a \mapsto Y(a, z)=\sum_{n \in \mathbb{Z}} a_{(n)} z^{-n-1} \quad\left(\text { where } a_{(n)} \in \operatorname{End} V\right),
\end{gathered}
$$

such that the following axioms hold:

(V1) For any $a, b \in V, \quad a_{(n)} b=0$ for $n$ sufficiently large.

(V2) $[D, Y(a, z)]=Y(D(a), z)=\frac{d}{d z} Y(a, z)$ for any $a \in V$.

(V3) $Y(\mathbb{1}, z)=\operatorname{Id}_{V} z^{0}$.

(V4) $Y(a, z) \mathbb{1} \in V[[z]]$ and $\left.Y(a, z) \mathbb{1}\right|_{z=0}=a$ for any $a \in V$ (self-replication).

(V5) For any $a, b \in V$, the fields $Y(a, z)$ and $Y(b, z)$ are mutually local; that is,

$$
(z-w)^{n}[Y(a, z), Y(b, w)]=0, \quad \text { for } n \text { sufficiently large. }
$$

A vertex superalgebra $V$ is called a vertex operator superalgebra (VOA) if, in addition, $V$ contains a vector $\omega$ (Virasoro element) such that

(V6) The components $L_{n}=\omega_{(n+1)}$ of the field

$$
Y(\omega, z)=\sum_{n \in \mathbb{Z}} \omega_{(n)} z^{-n-1}=\sum_{n \in \mathbb{Z}} L_{n} z^{-n-2}
$$

satisfy the Virasoro algebra relations

$$
\left[L_{n}, L_{m}\right]=(n-m) L_{n+m}+\delta_{n,-m} n^{3}-\frac{n}{12} C_{V i r},
$$

where $C_{V i r}$ acts on $V$ by a scalar, called the rank of $V$.

(V7) $D=L_{-1}$.

(V8) The operator $L_{0}$ is diagonalizable on $V$.

This completes the definition of a VOA.

As a consequence of the axioms of the vertex superalgebra we have the following important commutator formula:

$$
\left[Y\left(a, z_{1}\right), Y\left(b, z_{2}\right)\right]=\sum_{n \geq 0} \frac{1}{n !} Y\left(a_{(n)} b, z_{2}\right)\left[z_{1}^{-1}\left(\frac{\partial}{\partial z_{2}}\right)^{n} \delta\left(\frac{z_{2}}{z_{1}}\right)\right] .
$$


As usual, the delta function is

$$
\delta(z)=\sum_{n \in \mathbb{Z}} z^{n}
$$

By (V1), the sum on the right hand side of the commutator formula is actually finite. The commutator on the left hand side of (5.2) is of course the supercommutator.

Let us recall the definition of a normally ordered product of two fields. For a formal field $a(z)=\sum_{j \in \mathbb{Z}} a_{(j)} z^{-j-1}$ define its positive and negative parts as follows:

$$
a(z)_{-}=\sum_{j=0}^{\infty} a_{(j)} z^{-j-1}, \quad a(z)_{+}=\sum_{j=-1}^{-\infty} a_{(j)} z^{-j-1} .
$$

Then the normally ordered product of two formal fields $a(z), b(z)$ of parities $p(a), p(b)$ $\in\{0,1\}$ respectively, is defined as

$$
: a(z) b(z):=a(z)_{+} b(z)+(-1)^{p(a) p(b)} b(z) a(z)_{-} .
$$

The following property of vertex superalgebras will be used extensively in this paper:

$$
Y\left(a_{(-1)} b, z\right)=: Y(a, z) Y(b, z):, \quad \text { for all } a, b \in V .
$$

\section{Vertex Lie superalgebras}

An important source of vertex superalgebras is provided by vertex Lie superalgebras. In presenting this construction we will be following [5] (see also [8], 12], [25], 26]).

Let $\mathcal{L}$ be a Lie superalgebra with basis $\left\{u_{(n)}, c_{(-1)} \mid u \in \mathcal{U}, c \in \mathcal{C}, n \in \mathbb{Z}\right\}(\mathcal{U}, \mathcal{C}$ are some index sets). Define the corresponding fields in $\mathcal{L}\left[\left[z, z^{-1}\right]\right]$ :

$$
u(z)=\sum_{n \in \mathbb{Z}} u_{(n)} z^{-n-1}, \quad c(z)=c_{(-1)} z^{0}, \quad u \in \mathcal{U}, c \in \mathcal{C} .
$$

Let $\mathcal{F}$ be a subspace in $\mathcal{L}\left[\left[z, z^{-1}\right]\right]$ spanned by all the fields $u(z), c(z)$ and their derivatives of all orders.

Definition 6.1. A Lie superalgebra $\mathcal{L}$ with a basis as above is called a vertex Lie superalgebra if the following two conditions hold:

(VL1) for all $x, y \in \mathcal{U}$,

$$
\left[x\left(z_{1}\right), y\left(z_{2}\right)\right]=\sum_{j=0}^{n} f_{j}\left(z_{2}\right)\left[z_{1}^{-1}\left(\frac{\partial}{\partial z_{2}}\right)^{j} \delta\left(\frac{z_{2}}{z_{1}}\right)\right],
$$

where $f_{j}(z) \in \mathcal{F}, n \geq 0$ and depend on $x, y$.

(VL2) for all $c \in \mathcal{C}$, the elements $c_{(-1)}$ are central in $\mathcal{L}$.

Let $\mathcal{L}_{(+)}$be a subspace in $\mathcal{L}$ with basis $\left\{u_{(n)} \mid u \in \mathcal{U}, n \geq 0\right\}$ and let $\mathcal{L}_{(-)}$be a subspace with basis $\left\{u_{(n)}, c_{(-1)} \mid u \in \mathcal{U}, c \in \mathcal{C}, n<0\right\}$. Then $\mathcal{L}=\mathcal{L}_{(+)} \oplus \mathcal{L}_{(-)}$and $\mathcal{L}_{(+)}, \mathcal{L}_{(-)}$are in fact subalgebras in $\mathcal{L}$.

The universal enveloping vertex algebra $V_{\mathcal{L}}$ of a vertex Lie superalgebra $\mathcal{L}$ is defined as the induced module

$$
V_{\mathcal{L}}=\operatorname{Ind}_{\mathcal{L}_{(+)}}^{\mathcal{L}}(\mathbb{C} \mathbb{1})=U\left(\mathcal{L}_{(-)}\right) \otimes \mathbb{1},
$$

where $\mathbb{C} \mathbb{1}$ is a trivial 1-dimensional $\mathcal{L}_{(+)}$-module. 
Theorem 6.2 ([5], Theorem 4.8). Let $\mathcal{L}$ be a vertex Lie superalgebra. Then

(a) $V_{\mathcal{L}}$ has the structure of a vertex superalgebra with vacuum vector $\mathbb{1}$, infinitesimal translation $D$ being a natural extension of the derivation of $\mathcal{L}$ given by $D\left(u_{(n)}\right)$ $=-n u_{(n-1)}, D\left(c_{(-1)}\right)=0, u \in \mathcal{U}, c \in \mathcal{C}$, and the state-field correspondence map $Y$ defined by the formula

$$
\begin{gathered}
Y\left(a_{\left(-1-n_{1}\right)}^{1} \ldots a_{\left(-1-n_{k-1}\right)}^{k-1} a_{\left(-1-n_{k}\right)}^{k} \mathbb{1}, z\right) \\
=:\left(\frac{1}{n_{1} !}\left(\frac{\partial}{\partial z}\right)^{n_{1}} a^{1}(z)\right) \ldots \\
:\left(\frac{1}{n_{k-1} !}\left(\frac{\partial}{\partial z}\right)^{n_{k-1}} a^{k-1}(z)\right)\left(\frac{1}{n_{k} !}\left(\frac{\partial}{\partial z}\right)^{n_{k}} a^{k}(z)\right): \ldots:,
\end{gathered}
$$

where $a^{j} \in \mathcal{U}, n_{j} \geq 0$ or $a^{j} \in \mathcal{C}, n_{j}=0$.

(b) Any bounded $\mathcal{L}$-module is a vertex superalgebra module for $V_{\mathcal{L}}$.

(c) For an arbitrary character $\chi: \mathcal{C} \rightarrow \mathbb{C}$, the quotient module

$$
V_{\mathcal{L}}(\chi)=U\left(\mathcal{L}_{(-)}\right) \mathbb{1} / U\left(\mathcal{L}_{(-)}\right)\left\langle\left(c_{(-1)}-\chi(c)\right) \mathbb{1}\right\rangle_{c \in \mathcal{C}}
$$

is a quotient vertex superalgebra.

(d) Any bounded $\mathcal{L}$-module in which $c_{(-1)}$ act as $\chi(c) \mathrm{Id}$, for all $c \in \mathcal{C}$, is a vertex superalgebra module for $V_{\mathcal{L}}(\chi)$.

The value $\chi(c)$ is referred to as a central charge or level.

The vertex algebra that controls the representation theory of $\mathcal{D} \ltimes \mathcal{K}$ is the tensor product of three VOAs: a subalgebra $V_{H y p}^{+}$of a hyperbolic lattice vertex algebra, an affine $\widehat{g l}_{N}$ vertex algebra $V_{g l_{N}}$ at level 1, and the Virasoro vertex algebra $V_{V i r}$ of rank 0 . In order to apply the results of [2] to the representation theory of $\mathcal{D} \ltimes \mathcal{K}$, we use specializations $\dot{\mathfrak{g}}=(0)$ and $\tau=0$. In this specialization one has to fix the following values for the various central charges appearing in [2], Theorems 5.3 and 6.4:

$$
\begin{gathered}
c=1, \quad c_{s l_{N}}=1, \quad c_{H e i}=N, \\
c_{V H}=\frac{N}{2}, \quad c_{V i r}^{\prime}=0 .
\end{gathered}
$$

Let us briefly review the constructions of these three vertex algebras.

Hyperbolic lattice VOA. Consider a hyperbolic lattice Hyp, which is a free abelian group on $2 N$ generators $\left\{u^{a}, v^{a} \mid a=1, \ldots, N\right\}$ with the symmetric bilinear form

$$
(\cdot \mid \cdot): \operatorname{Hyp} \times \operatorname{Hyp} \rightarrow \mathbb{Z},
$$

defined by

$$
\left(u^{a} \mid v^{b}\right)=\delta_{a b}, \quad\left(u^{a} \mid u^{b}\right)=\left(v^{a} \mid v^{b}\right)=0, \quad a, b=1, \ldots, N .
$$

We complexify Hyp to get a $2 N$-dimensional vector space

$$
\mathcal{H}=\operatorname{Hyp} \otimes_{\mathbb{Z}} \mathbb{C}
$$

and extend the bilinear form by linearity on $\mathcal{H}$. Next, we affinize $\mathcal{H}$ by defining a Heisenberg Lie algebra

$$
\widehat{\mathcal{H}}=\mathbb{C}\left[t, t^{-1}\right] \otimes \mathcal{H} \oplus \mathbb{C} K
$$


with Lie bracket

$$
\begin{gathered}
{\left[x_{(n)}, y_{(m)}\right]=n(x \mid y) \delta_{n,-m} K, \quad x, y \in \mathcal{H},} \\
{[K, \widehat{\mathcal{H}}]=0 .}
\end{gathered}
$$

Here and in what follows, we are using the notation $x_{(n)}=t^{n} \otimes x$.

The algebra $\widehat{\mathcal{H}}$ has a triangular decomposition $\widehat{\mathcal{H}}=\widehat{\mathcal{H}}_{-} \oplus \widehat{\mathcal{H}}_{0} \oplus \widehat{\mathcal{H}}_{+}$, where $\widehat{\mathcal{H}}_{0}=1 \otimes \mathcal{H} \oplus \mathbb{C} K$ and $\widehat{\mathcal{H}}_{ \pm}=t^{ \pm 1} \mathbb{C}\left[t^{ \pm 1}\right] \otimes \mathcal{H}$.

Let $\mathrm{Hyp}^{+}$be an isotropic sublattice of Hyp generated by $\left\{u^{a} \mid a=1, \ldots, N\right\}$. We consider its group algebra $\mathbb{C}\left[\mathrm{Hyp}^{+}\right]=\mathbb{C}\left[e^{ \pm u^{1}}, \ldots, e^{ \pm u^{N}}\right]$ and define the action of $\widehat{\mathcal{H}}_{0} \oplus \widehat{\mathcal{H}}_{+}$on $\mathbb{C}\left[\mathrm{Hyp}^{+}\right]$by

$$
x_{(0)} e^{y}=(x \mid y) e^{y}, K e^{y}=e^{y}, \widehat{\mathcal{H}}_{+} e^{y}=0 .
$$

To be consistent with our previous notation, we set $q_{a}=e^{u^{a}}, a=1, \ldots, N$.

Finally, let $V_{H y p}^{+}$be the induced module

$$
V_{H y p}^{+}=\operatorname{Ind}_{\widehat{\mathcal{H}}_{0} \oplus \widehat{\mathcal{H}}_{+}}^{\widehat{\mathcal{C}}}\left(\mathbb{C}\left[\mathrm{Hyp}^{+}\right]\right) \text {. }
$$

We coordinatize $V_{H y p}^{+}$as a Fock space over $\widehat{\mathcal{H}}$ :

$$
V_{H y p}^{+}=\mathbb{C}\left[q_{1}^{ \pm 1}, \ldots, q_{N}^{ \pm 1}\right] \otimes \mathbb{C}\left[u_{p j},\left.v_{p j}\right|_{j=1,2, \ldots} ^{p=1, \ldots, N}\right],
$$

where $\widehat{\mathcal{H}}$ acts by operators of multiplication and differentiation:

$$
\begin{gathered}
u_{(-j)}^{p}=j u_{p j}, \quad u_{(j)}^{p}=\frac{\partial}{\partial v_{p j}}, \quad u_{(0)}^{p}=0, \\
v_{(-j)}^{p}=j v_{p j}, \quad v_{(j)}^{p}=\frac{\partial}{\partial u_{p j}}, \quad v_{(0)}^{p}=q_{p} \frac{\partial}{\partial q_{p}},
\end{gathered}
$$

for $p=1, \ldots, N, j=1,2, \ldots$

The space $V_{H y p}^{+}$has the structure of a vertex algebra - it is a vertex subalgebra in the vertex algebra corresponding to lattice Hyp. Here we give the values of the state-field correspondence map on the generators of this vertex algebra:

$$
\begin{gathered}
Y\left(u_{p 1}, z\right)=u^{p}(z)=\sum_{j \in \mathbb{Z}} u_{(j)}^{p} z^{-j-1} \\
Y\left(v_{p 1}, z\right)=v^{p}(z)=\sum_{j \in \mathbb{Z}} v_{(j)}^{p} z^{-j-1}, p=1, \ldots, N \\
Y\left(q^{r}, z\right)=q^{r} \exp \left(\sum_{p=1}^{N} r_{p} \sum_{j=1}^{\infty} \frac{z^{j}}{j} u_{(-j)}^{p}\right) \exp \left(-\sum_{p=1}^{N} r_{p} \sum_{j=1}^{\infty} \frac{z^{-j}}{j} u_{(j)}^{p}\right) .
\end{gathered}
$$

The Virasoro element of $V_{H y p}^{+}$is

$$
\omega^{H y p}=\sum_{p=1}^{N} u_{p 1} v_{p 1}
$$

and the Virasoro field is

$$
Y\left(\omega^{H y p}, z\right)=\sum_{p=1}^{N}: u^{p}(z) v^{p}(z): .
$$

The rank of $V_{H y p}^{+}$is $2 N$. 
Fix $\gamma \in \mathbb{C}^{N}$. The space

$$
M_{H y p}(\gamma)=q^{\gamma} \mathbb{C}\left[q_{1}^{ \pm 1}, \ldots, q_{N}^{ \pm 1}\right] \otimes \mathbb{C}\left[u_{p j},\left.v_{p j}\right|_{j=1,2, \ldots} ^{p=1, \ldots, N}\right]
$$

has a natural structure of a simple module for $V_{H y p}^{+}$(see e.g. [2] for details).

Affine $\widehat{g l}_{N} V O A$. The second vertex algebra that we will need is the affine $\widehat{g l}_{N}$ vertex algebra at level 1 . Since $g l_{N}$ is reductive, but not simple, it has more than one affinization. Here we consider a particular version of $\widehat{g l}_{N}$ :

$$
\widehat{g l}_{N}=\mathbb{C}\left[t, t^{-1}\right] \otimes g l_{N} \oplus \mathbb{C} C
$$

with Lie bracket

$$
\left[t^{n} \otimes X, t^{m} \otimes Y\right]=t^{n+m} \otimes[X, Y]+n \delta_{n,-m} \operatorname{Tr}(X Y) C, \quad X, Y \in g l_{N} .
$$

We note that $\widehat{g l}_{N}$ is a vertex Lie algebra and consider its universal enveloping vertex algebra $V_{g l_{N}}$ at level 1 (i.e., $\chi(C)=1$ ).

Let us give the value of the state-field correspondence map on the generators of this affine vertex algebra:

$$
Y\left(X_{(-1)} \mathbb{1}, z\right)=X(z)=\sum_{j \in \mathbb{Z}} X_{(j)} z^{-j-1}, \quad \text { for } \quad X \in g l_{N} .
$$

Since $g l_{N}$ has a decomposition $g l_{N}=s l_{N} \oplus \mathbb{C} I$, where $I$ is the identity $N \times N$ matrix, the affine $\widehat{g l}_{N}$ vertex algebra is the tensor product of the affine $\widehat{s l}_{N}$ vertex algebra and a Heisenberg vertex algebra. The Virasoro element $\omega^{g l_{N}}$ of $V_{g l_{N}}$ can thus be written as a sum of the Virasoro elements $\omega^{s l_{N}}$ for the affine $\widehat{s l}_{N}$ vertex algebra and $\omega^{H e i}$ for the Heisenberg vertex algebra. The usual formula for the Virasoro element of an affine vertex algebra gives the following explicit expression:

$$
\omega^{s l_{N}}=\frac{1}{2(N+1)}\left(\sum_{i, j=1}^{N} E_{(-1)}^{i j} E_{(-1)}^{j i} \mathbb{1}-\frac{1}{N} I_{(-1)} I_{(-1)} \mathbb{1}\right) .
$$

The rank of the affine $\widehat{s l}_{N}$ vertex algebra at level 1 is $N-1$.

For the Heisenberg vertex algebra we choose a non-standard Virasoro element (see [2], (4.33)):

$$
\omega^{H e i}=\frac{1}{2 N} I_{(-1)} I_{(-1)} \mathbb{1}+\frac{1}{2} I_{(-2)} \mathbb{1} .
$$

The rank of this Heisenberg VOA is $1-3 N$.

Adding these two Virasoro elements, we get the Virasoro element for $V_{g l_{N}}$ :

$$
\omega^{g l_{N}}=\frac{1}{2(N+1)}\left(\sum_{i, j=1}^{N} E_{(-1)}^{i j} E_{(-1)}^{j i} \mathbb{1}+I_{(-1)} I_{(-1)} \mathbb{1}\right)+\frac{1}{2} I_{(-2)} \mathbb{1} .
$$

The corresponding Virasoro field is

$$
Y\left(\omega^{g l_{N}}, z\right)=\frac{1}{2(N+1)}\left(\sum_{i, j=1}^{N}: E^{i j}(z) E^{j i}(z):+: I(z) I(z):\right)+\frac{1}{2} \frac{d}{d z} I(z) .
$$

The rank of $V_{g l_{N}}$ is $-2 N$.

Let $W$ be a finite-dimensional simple module for $g l_{N}$. Let $C$ act on $W$ as the identity operator and set $\left(t \mathbb{C}[t] \otimes g l_{N}\right) W=0$. Construct the generalized Verma module for the Lie algebra $\widehat{g l}_{N}$ as the induced module from $W$, and consider its 
irreducible quotient $L_{g l_{N}}(W)$. Then $L_{g l_{N}}(W)$ is a simple module for the vertex algebra $V_{g l_{N}}$.

Virasoro VOA. The last vertex algebra that we need to introduce is the Virasoro vertex algebra $V_{V i r}$ of rank 0 . The Virasoro Lie algebra (5.1) is a vertex Lie algebra with $\mathcal{U}=\left\{\omega^{\text {Vir }}\right\}$ and $\mathcal{C}=\left\{C_{V i r}\right\}$, where

$$
\omega^{\operatorname{Vir}}(z)=\sum_{j \in \mathbb{Z}} \omega^{\operatorname{Vir}}{ }_{(j)} z^{-j-1}=\sum_{j \in \mathbb{Z}} L_{j} z^{-j-2} .
$$

Let $V_{V i r}$ be its universal enveloping vertex algebra of zero central charge, $\chi\left(C_{V i r}\right)=$ 0 .

Let $L_{V i r}(h)$ be the irreducible highest weight module for the Virasoro Lie algebra with central charge 0 with highest weight vector $\mathrm{v}_{h}$, satisfying $L_{0} \mathrm{v}_{h}=h \mathrm{v}_{h}$.

The vertex algebra that controls the representation theory of $\mathcal{D} \ltimes \mathcal{K}$ is the tensor product of the sub-VOA $V_{H y p}^{+}$of the hyperbolic lattice vertex algebra, affine $\widehat{g l}_{N}$ vertex algebra $V_{g l_{N}}$ at level 1, and the Virasoro VOA $V_{V i r}$ of rank 0 ,

$$
V_{H y p}^{+} \otimes V_{g l_{N}} \otimes V_{V i r}
$$

with the Virasoro element

$$
\omega=\omega^{H y p}+\omega^{g l_{N}}+\omega^{V i r} .
$$

The rank of this VOA is $2 N-2 N+0=0$. Now we are ready to present a result of 2] (Theorems 5.3 and 6.4):

Theorem 6.3 ([2]). (i) Let $M_{H y p}, M_{g l_{N}}$ and $M_{V i r}$ be modules for $V_{H y p}^{+}, V_{g l_{N}}$ and $V_{V i r}$ respectively. Then the tensor product

$$
\mathcal{M}=M_{H y p} \otimes M_{g l_{N}} \otimes M_{V i r}
$$

is a module for the Lie algebra $\mathcal{D} \ltimes \mathcal{K}$ with the action given as follows:

$$
\begin{gathered}
\sum_{j \in \mathbb{Z}} t_{0}^{j} t^{r} k_{0} z^{-j}=k_{0}(r, z) \mapsto Y\left(q^{r}, z\right), \\
\sum_{j \in \mathbb{Z}} t_{0}^{j} t^{r} k_{a} z^{-j-1}=k_{a}(r, z) \mapsto u^{a}(z) Y\left(q^{r}, z\right),
\end{gathered}
$$

$$
\sum_{j \in \mathbb{Z}} t_{0}^{j} t^{r} d_{a} z^{-j-1}=d_{a}(r, z) \mapsto: v^{a}(z) Y\left(q^{r}, z\right):+\sum_{p=1}^{N} r_{p} E^{p a}(z) Y\left(q^{r}, z\right)
$$$$
\sum_{j \in \mathbb{Z}} t_{0}^{j} t^{r} d_{0} z^{-j-2}=d_{0}(r, z) \mapsto-: Y(\omega, z) Y\left(q^{r}, z\right):-\sum_{i, j=1}^{N} r_{i} u^{j}(z) E^{i j}(z) Y\left(q^{r}, z\right)
$$

$$
+\sum_{p=1}^{N} r_{p}\left(\frac{d}{d z} u^{p}(z)\right) Y\left(q^{r}, z\right)
$$

for $a=1, \ldots, N$.

(ii) The module

$$
L(W, \gamma, h)=M_{H y p}(\gamma) \otimes L_{g l_{N}}(W) \otimes L_{V i r}(h)
$$

is an irreducible module over the Lie algebra $\mathcal{D} \ltimes \mathcal{K}$. 


\section{Generalized Wakimoto modules}

In 3 the structure of irreducible modules $L(T)$ over the Lie algebra of vector fields was determined in the case of a 2-dimensional torus $(N=1)$. It turned out that the situation was analogous to the case of a basic module for an affine Kac-Moody algebra, which remains irreducible when restricted to the principal Heisenberg subalgebra [16], [13. For the Lie algebra of vector fields on $\mathbb{T}^{2}$ this role is played by its loop subalgebra $\widetilde{s l}_{2}=\mathbb{C}\left[t_{0}, t_{0}^{-1}\right] \otimes s l_{2}$. Indeed we have $s l_{2}$ embedded into Vect $\mathbb{T}^{1}$ :

$$
s l_{2} \cong\left\langle t_{1}^{-1} d_{1}, d_{1}, t_{1} d_{1}\right\rangle \subset \mathbb{C}\left[t_{1}, t_{1}^{-1}\right] d_{1} .
$$

This extends to an embedding

$$
\widetilde{s l}_{2} \cong \mathbb{C}\left[t_{0}, t_{0}^{-1}\right] \otimes\left\langle t_{1}^{-1} d_{1}, d_{1}, t_{1} d_{1}\right\rangle \subset \operatorname{Vect} \mathbb{T}^{2} .
$$

The following theorem was proved in 3 :

Theorem $7.1([3])$. Let $W$ be the 1-dimensional $g l_{1}$-module in which the identity matrix acts as multiplication by $\alpha \in \mathbb{C}$. Assume $\alpha \notin \mathbb{Q}, \beta=\frac{\alpha(\alpha-1)}{2}$, and let $\gamma \in \mathbb{C}$. Then the module $L(T)=L(\alpha, \beta, \gamma)$ over the Lie algebra Vect $\mathbb{T}^{2}$ remains irreducible when restricted to the subalgebra $\widetilde{s l}{ }_{2}$.

Note that 3 uses a different convention for the sign of $\alpha$.

The loop algebra $\widetilde{s l}_{2}=\mathbb{C}\left[t_{0}, t_{0}^{-1}\right] \otimes s l_{2}$ is $\mathbb{Z}$-graded by degree in $t_{0}$. This gives

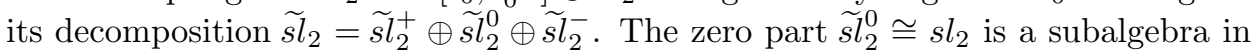
$\mathbb{C}\left[t_{1}, t_{1}^{-1}\right] d_{1}$ and thus acts on $T$. The positive part $\widetilde{s l}_{2}^{+}$acts on $T$ trivially. We can form the generalized Verma module over $\widetilde{s l}_{2}$ :

$$
\operatorname{Ind}_{\widetilde{s l}_{2}^{0} \oplus \widetilde{s} l_{2}^{+}}^{\widetilde{s}_{2}} T(\alpha, \beta, \gamma) \cong U(\widetilde{s l}-\overline{2}) \otimes T(\alpha, \beta, \gamma) .
$$

By the results of [11], this generalized Verma module is irreducible over $\widetilde{s l}_{2}$ if and only if $\alpha \notin \frac{1}{2} \mathbb{Z}$. This gives the following:

Corollary 7.2 ([3]). Let $\alpha \in \mathbb{C}, \alpha \notin \mathbb{Q}, \beta=\frac{\alpha(\alpha-1)}{2}$. Then the Vect $\mathbb{T}^{2}$-module $L(\alpha, \beta, \gamma)$ when restricted to $\widetilde{s l}_{2}$ is isomorphic to the generalized Verma module over $\widetilde{s l}_{2}$ :

$$
L(\alpha, \beta, \gamma) \cong U\left(\widetilde{s l}_{2}^{-}\right) \otimes T(\alpha, \beta, \gamma) .
$$

These results show that the loop subalgebra $\widetilde{s l}_{2}$ plays a crucial role in the representation theory of the Lie algebra of vector fields on $\mathbb{T}^{2}$. It is natural to conjecture that in the representation theory of $\mathcal{D}=\operatorname{Vect} \mathbb{T}^{N+1}$ such a role is played by the loop algebra $\widetilde{s l}_{N+1}$. Indeed, $\mathcal{D}_{0}$ has $\operatorname{VectT}^{N}$ as a subalgebra and Vect $\mathbb{T}^{N}$ contains $s l_{N+1}$. The embedding of $s l_{N+1}$ into $\operatorname{VectT}^{N}$ is the following: $e_{i j} \mapsto t_{i} t_{j}^{-1} d_{j}$, $e_{i, N+1} \mapsto-t_{i} D, e_{N+1, i} \mapsto t_{i}^{-1} d_{i}, e_{N+1, N+1} \mapsto-D$, where $D=\sum_{k=1}^{N} d_{k}, i, j \leq N$ (see e.g. 22]). Thus Vect $\mathbb{T}^{N+1}$ contains the loop algebra $\widetilde{s} l_{N+1}=\mathbb{C}\left[t_{0}, t_{0}^{-1}\right] \otimes s l_{N+1}$.

Let

$$
s l_{N+1}=s l_{N+1}^{-} \oplus \mathfrak{h} \oplus s l_{N+1}^{+}
$$

be the standard triangular decomposition of $s l_{N+1}$ with a Cartan subalgebra $\mathfrak{h}$ generated by $d_{1}, \ldots, d_{N}$. Set

$$
\widetilde{s} l_{N+1}^{ \pm}=t_{0}^{ \pm} \mathbb{C}\left[t_{0}^{ \pm}\right] \otimes s l_{N+1} .
$$


Then $\mathfrak{p}=s l_{N+1} \oplus \widetilde{s l_{N+1}^{+}}$is a parabolic subalgebra of $\widetilde{s l}_{N+1}$. The modules $T(W, \beta, \gamma)$ may be viewed as $s l_{N+1}$-modules and as a $\mathfrak{p}$-module with a trivial action of $\widetilde{s l} l_{N+1}^{+}$. Hence we can form the generalized Verma module over $\widetilde{s l}_{N+1}$ :

$$
U\left(\widetilde{s l}_{N+1}\right) \otimes_{U(\mathfrak{p})} T(W, \beta, \gamma) \cong U\left(\widetilde{s l}_{N+1}^{-}\right) \otimes T(W, \beta, \gamma) .
$$

Note that there is an action of $d_{0}$ on this module.

It turns out, however, that in general, the action of $\widetilde{s l}_{N+1}$ on the generalized Verma module cannot be extended to the action of the bigger algebra $\operatorname{Vect} \mathbb{T}^{N+1}$. Instead one should use certain generalized Wakimoto modules. We define the generalized Wakimoto modules in the following way:

Definition 7.3. Let $T$ be a weight $s l_{N+1}$-module with finite-dimensional weight spaces (with respect to $\mathfrak{h}$ ). Let $d_{0}$ act on $T$ as a scalar operator. A generalized Wakimoto module $\mathcal{M}$ with top $T$ is an $\widetilde{s l}_{N+1} \oplus \mathbb{C} d_{0}$-module that contains $T$ as an $s l_{N+1} \oplus \mathbb{C} d_{0}$-submodule with $\widetilde{s l}{ }_{N+1}^{+}$acting on $T$ trivially and having the property that the character of $\mathcal{M}$ with respect to $\mathfrak{h} \oplus \mathbb{C} d_{0}$ coincides with the character of the generalized Verma module for $\widetilde{s l}_{N+1}$ :

$$
\operatorname{char} \mathcal{M}=\operatorname{char}\left(U\left(\widetilde{s l}_{N+1}^{-}\right)\right) \times \operatorname{char} T .
$$

Here $\operatorname{char} U\left(\widetilde{s l}_{N+1}^{-}\right)$and $\operatorname{char} T$ are the characters of the corresponding weight modules, where $U\left(\widetilde{s l}_{N+1}^{-}\right)$is viewed as an $\widetilde{s l}_{N+1} \oplus \mathbb{C} d_{0}$-module with respect to the adjoint action. The generalized Verma module is by definition a generalized Wakimoto module. In the case when the generalized Verma module is irreducible, it is the only generalized Wakimoto module with the given top $T$. As we mentioned above, for $\widetilde{s l_{2}}$ this happens for the tops $T(\alpha, \beta, \gamma)$ with $\alpha \notin \frac{1}{2} \mathbb{Z}[11$. For $N>1$ and any top $T(W, \beta, \gamma)$ with a finite-dimensional $g l_{N}$-module $W$, the generalized Verma module over $\widetilde{s l}_{N+1}$ is always reducible.

We shall now see that Theorem 6.3 yields a construction of a generalized Wakimoto module for $\widetilde{s l}_{N+1}$. These modules admit the action of the whole algebra of vector fields on $\mathbb{T}^{N+1}$.

Proposition 7.4. Let $M_{g l_{N}}(W)$ be the generalized Verma module for $\widehat{g l}_{N}$ at level 1 , induced from an irreducible finite-dimensional $\mathrm{gl}_{N}$-module $W$. Then the module

$$
\mathcal{M}=q^{\gamma} \mathbb{C}\left[q_{1}^{ \pm 1}, \ldots, q_{N}^{ \pm 1}\right] \otimes \mathbb{C}\left[u_{p j}, v_{p j}\right] \otimes M_{g l_{N}}(W)
$$

is a generalized Wakimoto module for the loop algebra $\widetilde{s l}_{N+1}$.

Proof. Applying Theorem 6.3 with a trivial 1-dimensional Virasoro module, we see that $\mathcal{M}$ is a module for the Lie algebra $\operatorname{Vect} \mathbb{T}^{N+1}$. By restriction, view $\mathcal{M}$ as an $\widetilde{s l}_{N+1}$-module. The top of the module $\mathcal{M}$ is the tensor module

$$
T(W, \gamma)=q^{\gamma} \mathbb{C}\left[q_{1}^{ \pm 1}, \ldots, q_{N}^{ \pm 1}\right] \otimes W .
$$

To show that $\mathcal{M}$ is a generalized Wakimoto module for $\widetilde{s l}_{N+1}$, we need to compare the characters of $\mathbb{C}\left[u_{p j}, v_{p j}\right] \otimes U\left(\widehat{g l}_{N}^{-}\right)$and $U\left(\widetilde{s l}_{N+1}^{-}\right)$. We have

$\operatorname{char} \mathbb{C}\left[u_{p j}, v_{p j}\right] \times \operatorname{char} U\left(\widehat{g l}_{N}^{-}\right)=\prod_{k=1}^{\infty}\left(1-s^{k}\right)^{-2 N} \times \prod_{k=1}^{\infty}\left(1-s^{k}\right)^{-N^{2}}=\operatorname{char} U\left(\widetilde{s l}_{N+1}^{-}\right)$.

This completes the proof of the proposition. 
Theorem 6.3 describes irreducible $\mathcal{D} \ltimes \mathcal{K}$-modules. We would like to study their reductions to the subalgebra $\mathcal{D}$. In general, when reduced to a subalgebra, modules become reducible. Here, however, the link with generalized Wakimoto modules for $\widetilde{s l}_{N+1}$ and the result of [3] for $N=1$ give us hope that the situation may be better than one would expect a priori.

\section{Duality for modules over the Lie Algebra of Vector FieldS}

In this section we will establish a duality for the class of modules described in Theorem 6.3 (ii) that will be useful for the study of their irreducibility as modules over Lie algebra $\mathcal{D}$. We begin by looking at this question in a general setup.

Let $\mathcal{L}$ be a $\mathbb{Z}$-graded Lie algebra $\mathcal{L}=\bigoplus_{n \in \mathbb{Z}} \mathcal{L}_{n}$ with an anti-involution $\sigma$ such that $\sigma\left(\mathcal{L}_{n}\right)=\mathcal{L}_{-n}$. Extend $\sigma$ to the universal enveloping algebra $U(\mathcal{L})$ by $\sigma(a b)=$ $\sigma(b) \sigma(a)$. Let $\mathcal{L}_{ \pm}=\bigoplus_{n>0} \mathcal{L}_{ \pm n}$. Suppose $T_{1}, T_{2}$ are two $\mathcal{L}_{0}$-modules with a bilinear pairing

$$
T_{1} \times T_{2} \rightarrow \mathbb{C}
$$

such that

$$
\left\langle x w_{1}, w_{2}\right\rangle=\left\langle w_{1}, \sigma(x) w_{2}\right\rangle
$$

for all $x \in \mathcal{L}_{0}, w_{1} \in T_{1}, w_{2} \in T_{2}$.

For an $\mathcal{L}_{0}$-module $T$ we let $\mathcal{L}_{+}$act on $T$ trivially and construct the generalized Verma module for $\mathcal{L}: M(T)=U\left(\mathcal{L}_{-}\right) \otimes T$.

The generalized Verma module $M(T)$ inherits the $\mathbb{Z}$-grading (assuming the degree of $T$ to be zero). Define the radical of a generalized Verma module $M(T)$ as the maximal homogeneous submodule trivially intersecting with the top $T$. If $T$ is an irreducible $\mathcal{L}_{0}$-module, then the quotient $L(T)$ of $M(T)$ by its radical is an irreducible module for $\mathcal{L}$.

Consider the Shapovalov projection

$$
S: U(\mathcal{L}) \rightarrow U\left(\mathcal{L}_{0}\right)
$$

with kernel $\mathcal{L}_{-} U(\mathcal{L})+U(\mathcal{L}) \mathcal{L}_{+}$.

Define a bilinear pairing

$$
M\left(T_{1}\right) \times M\left(T_{2}\right) \rightarrow \mathbb{C}
$$

by

$$
\left\langle a w_{1}, b w_{2}\right\rangle=\left\langle w_{1}, S(\sigma(a) b) w_{2}\right\rangle
$$

for $a, b \in U\left(\mathcal{L}_{-}\right), w_{1} \in T_{1}, w_{2} \in T_{2}$.

Proposition 8.1. (i) The pairing (8.2) is contragredient, i.e.,

$$
\langle x u, v\rangle=\langle u, \sigma(x) v\rangle
$$

for all $x \in \mathcal{L}, u \in M\left(T_{1}\right), v \in M\left(T_{2}\right)$.

(ii) If $n \neq k$, then $\left\langle M\left(T_{1}\right)_{n}, M\left(T_{2}\right)_{k}\right\rangle=0$.

(iii) The radicals of $M\left(T_{1}\right), M\left(T_{2}\right)$ are in the kernel of the pairing.

(iv) Assume that $T_{1}, T_{2}$ are irreducible $\mathcal{L}_{0}$-modules and the pairing (8.1) is nonzero. Then the contragredient pairing factors to the simple modules

$$
L\left(T_{1}\right) \times L\left(T_{2}\right) \rightarrow \mathbb{C},
$$

on which it is non-degenerate. 
This proposition is standard (cf., 24, Proposition 2.8.1), and its proof is left to the reader as an exercise.

Next we will apply this proposition to establish the duality for the bounded modules described in Theorem 6.3 (ii).

We consider the following anti-involution on $\mathcal{D} \ltimes \mathcal{K}$ :

$$
\sigma\left(t_{0}^{j} t^{r} d_{a}\right)=t_{0}^{-j} t^{-r} d_{a}, \quad \sigma\left(t_{0}^{j} t^{r} k_{a}\right)=t_{0}^{-j} t^{-r} k_{a}, \quad a=0, \ldots, N .
$$

For a finite-dimensional simple $g l_{N}$-module $W$, on which the identity matrix $I$ acts as multiplication by $\alpha \in \mathbb{C}$, let $W^{*}$ be the dual space to $W$ with an $s l_{N}$-module structure of the dual module, but with $I$ acting as scalar $N-\alpha$. The natural pairing between $W$ and $W^{*}$ satisfies

$$
\left\langle E^{a b} w \mid w^{*}\right\rangle=\left\langle w \mid-E^{a b} w^{*}+\delta_{a b} w^{*}\right\rangle .
$$

Theorem 8.2. There exists a non-degenerate contragredient pairing of simple $\mathcal{D} \ltimes$ $\mathcal{K}$-modules defined in Theorem 6.3 (ii):

$$
L(W, \gamma, h) \times L\left(W^{*}, \gamma, h\right) \rightarrow \mathbb{C},
$$

satisfying

$$
\langle x u, v\rangle=\langle u, \sigma(x) v\rangle
$$

for all $x \in \mathcal{D} \ltimes \mathcal{K}, u \in L(W, \gamma, h), v \in L\left(W^{*}, \gamma, h\right)$.

For the proof of this theorem we will use an alternative construction of the simple $\mathcal{D} \ltimes \mathcal{K}$-module $L(W, \gamma, h)$ which is discussed in 2 . These modules may be abstractly defined using the approach of Theorem 3.1. The top of the module $L(W, \gamma, h)$ is the space

$$
T=T(W, \gamma, h)=q^{\gamma} \mathbb{C}\left[q_{1}^{ \pm 1}, \ldots, q_{N}^{ \pm 1}\right] \otimes W \otimes \mathrm{v}_{h},
$$

which is a module for the zero degree component $\mathcal{D}_{0} \ltimes \mathcal{K}_{0}$ of $\mathcal{D} \ltimes \mathcal{K}$ with respect to its $\mathbb{Z}$-grading by degree in $t_{0}$. The positive part of $\mathcal{D} \ltimes \mathcal{K}$ acts on $T(W, \gamma, h)$ trivially, and we can consider the induced $\mathcal{D} \ltimes \mathcal{K}$-module $M(T)$. The induced module has a unique irreducible quotient, which is isomorphic to $L(W, \gamma, h)$.

The action of $\mathcal{D}_{0} \ltimes \mathcal{K}_{0}$ on $T(W, \gamma, h)$ can be derived from Theorem 6.3 (i) (see Theorem 6.4 in [2] for details), and $T$ is essentially a tensor module that we discussed above:

$$
\begin{gathered}
t^{r} k_{0}\left(q^{\mu} \otimes w \otimes \mathrm{v}_{h}\right)=q^{\mu+r} \otimes w \otimes \mathrm{v}_{h}, \\
t^{r} k_{a}\left(q^{\mu} \otimes w \otimes \mathrm{v}_{h}\right)=0 \\
t^{r} d_{a}\left(q^{\mu} \otimes w \otimes \mathrm{v}_{h}\right)=\mu_{a} q^{\mu+r} \otimes w \otimes \mathrm{v}_{h}+\sum_{p=1}^{N} r_{p} q^{\mu+r} \otimes E^{p a} w \otimes \mathrm{v}_{h}, \\
t^{r} d_{0}\left(q^{\mu} \otimes w \otimes \mathrm{v}_{h}\right)=\beta q^{\mu+r} \otimes w \otimes \mathrm{v}_{h},
\end{gathered}
$$

where

$$
\beta=-h-\frac{\Omega_{W}}{2(N+1)}-\frac{\alpha(\alpha-N)}{2 N},
$$

$a=1, \ldots, N, r \in \mathbb{Z}^{N}, \mu \in \gamma+\mathbb{Z}^{N}$. Here $\Omega_{W}$ is the scalar with which the Casimir operator of $s l_{N}$ acts on $W$.

The claim of Theorem 8.2 follows from Proposition 8.1 and the following lemma. 
Lemma 8.3. Let $W$ be a simple finite-dimensional $g l_{N}$-module on which the identity matrix acts as scalar $\alpha$, and let $W^{*}$ be a gl $l_{n}$-module which is dual to $W$ as an $s l_{N}$-module, and on which the identity matrix acts as scalar $N-\alpha$. Then the pairing

$$
T(W, \gamma, h) \times T\left(W^{*}, \gamma, h\right) \rightarrow \mathbb{C}
$$

given by

$$
\left\langle q^{\mu} \otimes w \otimes \mathrm{v}_{h} \mid q^{\eta} \otimes w^{*} \otimes \mathrm{v}_{h}\right\rangle=\delta_{\mu, \eta}\left\langle w \mid w^{*}\right\rangle,
$$

is a non-degenerate contragredient pairing of $\mathcal{D}_{0} \ltimes \mathcal{K}_{0}$-modules.

Proof. Using (8.4) we get

$$
\begin{gathered}
\left\langle t^{r} k_{0}\left(q^{\mu} \otimes w \otimes \mathrm{v}_{h}\right) \mid q^{\eta} \otimes w^{*} \otimes \mathrm{v}_{h}\right\rangle=\delta_{\mu+r, \eta}\left\langle w \mid w^{*}\right\rangle \\
=\delta_{\mu, \eta-r}\left\langle w \mid w^{*}\right\rangle=\left\langle q^{\mu} \otimes w \otimes \mathrm{v}_{h} \mid \sigma\left(t^{r} k_{0}\right)\left(q^{\eta} \otimes w^{*} \otimes \mathrm{v}_{h}\right)\right\rangle .
\end{gathered}
$$

In the case of $t^{r} k_{a}, a=1, \ldots, N$, both left and right hand sides are zero. Let us verify the contragredient property for the action of $t^{r} d_{a}, a=1, \ldots, N$ :

$$
\begin{gathered}
\left\langle t^{r} d_{a}\left(q^{\mu} \otimes w \otimes \mathrm{v}_{h}\right) \mid q^{\eta} \otimes w^{*} \otimes \mathrm{v}_{h}\right\rangle \\
=\left\langle\mu_{a} q^{\mu+r} \otimes w \otimes \mathrm{v}_{h}+\sum_{p=1}^{N} r_{p} q^{\mu+r} \otimes E^{p a} w \otimes \mathrm{v}_{h} \mid q^{\eta} \otimes w^{*} \otimes \mathrm{v}_{h}\right\rangle \\
=\delta_{\mu+r, \eta}\left(\mu_{a}\left\langle w \mid w^{*}\right\rangle+\sum_{p=1}^{N} r_{p}\left\langle E^{p a} w \mid w^{*}\right\rangle\right) \\
=\delta_{\mu, \eta-r}\left(\mu_{a}\left\langle w \mid w^{*}\right\rangle+\sum_{p=1}^{N} r_{p}\left\langle w \mid-E^{p a} w^{*}+\delta_{p a} w^{*}\right\rangle\right) \\
=\delta_{\mu, \eta-r}\left(\left(\eta_{a}-r_{a}\right)\left\langle w \mid w^{*}\right\rangle-\sum_{p=1}^{N} r_{p}\left\langle w \mid E^{p a} w^{*}\right\rangle+r_{a}\left\langle w \mid w^{*}\right\rangle\right) \\
=\left\langle q^{\mu} \otimes w \otimes \mathrm{v}_{h} \mid \sigma\left(t^{r} d_{a}\right)\left(q^{\eta} \otimes w^{*} \otimes \mathrm{v}_{h}\right)\right\rangle .
\end{gathered}
$$

Finally, to check the case of $t^{r} d_{0}$, we note that the constant $\beta$ in (8.8) is the same for $T(W, \gamma, h)$ and $T\left(W^{*}, \gamma, h\right)$. This follows from the fact that the Casimir operator for $s l_{N}$ acts with the same scalar on $W$ and $W^{*}$, while the last term in (8.8) is invariant under the substitution $\alpha \mapsto N-\alpha$. Thus the computation in the case of $t^{r} d_{0}$ is analogous to the case of $t^{r} k_{0}$. This completes the proof of the lemma.

Remark 8.4. The pairing (8.3) is in fact a product of contragredient pairings of tensor factors

$M_{H y p}(\gamma) \times M_{H y p}(\gamma) \rightarrow \mathbb{C}, \quad L_{g l_{N}}(W) \times L_{g l_{N}}\left(W^{*}\right) \rightarrow \mathbb{C}, \quad L_{V i r}(h) \times L_{V i r}(h) \rightarrow \mathbb{C}$, with respect to appropriate anti-involutions of corresponding Lie algebras.

Remark 8.5. The duality of Theorem 8.2 can be alternatively constructed via the vertex algebra approach, using the definition of the contragredient module over a vertex algebra (see section 5.2 in [10]).

One of the goals of this paper is to analyze which of the modules defined in Theorem 6.3 (ii) remain irreducible after restriction to $\mathcal{D}$. First of all, let us look at the question of irreducibility of the top $T(W, \gamma, h)$ as a module over $\mathcal{D}_{0}$. 
Lemma 8.6. Let $W$ be an irreducible finite-dimensional $g l_{N}$-module. The module $T(W, \gamma, h)$ is reducible as a $\mathcal{D}_{0}$-module if and only if it is reducible as a $\operatorname{Vect}^{N}$ module (see Theorem 2.1) and $h=0$.

Proof. Clearly, if $T(W, \gamma, h)$ is reducible as a $\mathcal{D}_{0}$-module, it must also be reducible as a Vect $\mathbb{T}^{N}$-module. By Theorem 2.1, all such modules appear in the de Rham complex. Note that

$$
\mathcal{D}_{0}=\operatorname{Vect} \mathbb{T}^{N} \oplus \mathbb{C}\left[t_{1}^{ \pm 1}, \ldots, t_{N}^{ \pm 1}\right] d_{0},
$$

and by (8.7) $t^{r} d_{0}$ acts on $T(W, \gamma, h)$ as multiplication by $\beta q^{r}$. It is well known that in the modules of differential forms there are no proper submodules that are $\mathbb{C}\left[q_{1}^{ \pm 1}, \ldots, q_{N}^{ \pm 1}\right]$-invariant. Thus for $T(W, \gamma, h)$ to be reducible as a $\mathcal{D}_{0}$-module it is necessary and sufficient that it is reducible as a Vect $\mathbb{T}^{N}$-module and the value of $\beta$ given by (8.8) is zero. Let us analyze the values of $\beta$ for the modules in the de Rham complex. For the modules $\Omega^{0}\left(\mathbb{T}^{N}\right)$ and $\Omega^{N}\left(\mathbb{T}^{N}\right)$ the $s l_{N}$-module $W$ is trivial, so the Casimir operator acts with constant $\Omega_{W}=0$, while the identity matrix acts on $W$ with scalars $\alpha=0$ and $\alpha=N$ respectively. Simplifying the expression in (8.8) we get in this case that $\beta=-h$. In the case of the modules of $k$-forms $\Omega^{k}\left(\mathbb{T}^{N}\right), k=1, \ldots, N-1$, the highest weight of the corresponding $s l_{N^{-}}$ module $W$ is the fundamental weight $\omega_{k}$. A standard computation shows that in this case the Casimir operator acts with the scalar

$$
\Omega_{W}=\left(\omega_{k} \mid \omega_{k}+2 \rho\right)=k(N-k) \frac{N+1}{N} .
$$

Since the identity matrix acts with the scalar $\alpha=k$, the formula (8.8) again simplifies to $\beta=-h$. This implies the claim of the lemma.

Now consider an irreducible $\mathcal{D} \ltimes \mathcal{K}$-module $L(W, \gamma, h)$ described in Theorem 6.3 (ii), and assume that its top $T(W, \gamma, h)$ is irreducible as a $\mathcal{D}_{0}$-module. To show that $L(W, \gamma, h)$ remains irreducible as a module over $\mathcal{D}$, it is sufficient to establish two properties:

(C) Every critical vector of $L(W, \gamma, h)$ (i.e., annihilated by $\left.\mathcal{D}_{+}\right)$belongs to its top $T(W, \gamma, h)$.

(G) $L(W, \gamma, h)$ is generated by its top $T(W, \gamma, h)$ as a module over $\mathcal{D}_{-}$.

The following standard observation will be quite useful:

Lemma 8.7. Condition $(C)$ holds for the module $L(W, \gamma, h)$ if and only if condition $(G)$ holds for $L\left(W^{*}, \gamma, h\right)$.

This lemma is an immediate consequence of Theorem 8.2 , and we omit its proof.

Lemma 8.7 reduces the question of irreducibility of the family of modules $L(W, \gamma, h)$ to the question of existence of critical vectors. If both $L(W, \gamma, h)$ and $L\left(W^{*}, \gamma, h\right)$ satisfy condition $(\mathrm{C})$, then they are both irreducible as modules over $\mathcal{D}$.

\section{CRitical vectors}

In this section we will establish a necessary condition for the existence of nontrivial critical vectors in the modules $L(W, \gamma, h)$, which together with Lemma 8.7 will give a sufficient condition for the irreducibility of such modules. 
Theorem 9.1. Let $W$ be an irreducible finite-dimensional $g l_{N}$-module, $\gamma \in \mathbb{C}^{N}, h \in$ $\mathbb{C}$. Every critical vector (i.e., annihilated by $\mathcal{D}_{+}$) in the module $L(W, \gamma, h)$ belongs to its top, unless $h=0$ and $W$ is either a trivial sl $l_{N}$-module with identity matrix acting with scalar $\alpha=N-m N, m=1,2, \ldots$, or the highest weight of $W$ is a fundamental weight $\omega_{k}, k=1, \ldots, N-1$, with identity matrix acting by scalar $\alpha=k-m N, m=1,2, \ldots$

We will call a module $L(W, \gamma, h)$ exceptional if $h=0$, the identity matrix acts on $W$ by an integer $k \in \mathbb{Z}$ and $W$ is a trivial 1-dimensional $s l_{N}$-module when $k=0$ $\bmod N$ or has a fundamental highest weight $\omega_{k^{\prime}}$ with $1 \leq k^{\prime} \leq N-1$ and $k=k^{\prime}$ $\bmod N$.

Theorem 9.2. Let $W$ be an irreducible finite-dimensional $g l_{N}$-module, $\gamma \in \mathbb{C}^{N}, h \in$ $\mathbb{C}$. Every non-exceptional module $L(W, \gamma, h)$ is irreducible as a $\operatorname{Vect}^{N}$-module.

Theorem 9.2 is an immediate consequence of Theorem 9.1 and Lemma 8.7. The proof of Theorem 9.1 will be split into a sequence of lemmas.

Lemma 9.3. Let $g \in L(W, \gamma, h)$ be a critical vector. Then $g$ does not depend on variables $\left\{u_{p j}\right\}$, i.e., $g$ belongs to the subspace

$$
q^{\gamma} \mathbb{C}\left[q_{1}^{ \pm 1}, \ldots, q_{N}^{ \pm 1}\right] \otimes \mathbb{C}\left[\left.v_{p j}\right|_{j=1,2, \ldots} ^{p=1, \ldots, N}\right] \otimes L_{g l_{N}}(W) \otimes L_{V i r}(h) .
$$

Proof. The algebra $\mathcal{D}_{+}$contains the elements $t_{0}^{j} d_{p}$ with $p=1, \ldots, N, j \geq 1$, which act as $\frac{\partial}{\partial u_{p j}}$. The condition $\left(t_{0}^{j} d_{p}\right) g=0$ implies the claim of the lemma.

For a formal series $a(z)$ we denote by $a(z)_{-}$its part that only involves negative powers of $z$. Recalling that $d_{a}(r, z)=\sum_{j \in \mathbb{Z}} t_{0}^{j} t^{r} d_{a} z^{-j-1}$, we have $\left(z d_{a}(r, z)\right)_{-} g=0$. Using (6.11) for the action of $d_{a}(r, z)$ and taking into account that $g$ does not depend on $\left\{u_{p j}\right\}$, we get

$$
\begin{gathered}
q^{r}\left(\exp \left(\sum_{p=1}^{N} r_{p} \sum_{j=1}^{\infty} u_{p j} z^{j}\right)\left(\sum_{i=1}^{\infty} i v_{a i} z^{i}+q_{a} \frac{\partial}{\partial q_{a}}+\sum_{p=1}^{N} r_{p} \sum_{k \in \mathbb{Z}} E_{(k)}^{p a} z^{-k}\right)\right. \\
\left.\times \exp \left(-\sum_{p=1}^{N} r_{p} \sum_{j=1}^{\infty} \frac{z^{-j}}{j} \frac{\partial}{\partial v_{p j}}\right)\right)_{-} g=0 .
\end{gathered}
$$

Let us project to the subspace (9.1), setting $u_{p j}=0$ in the above equality. Also, since the operator of multiplication by $q^{r}$ is invertible, we can drop it. We then get

$$
P_{a}(r, z)_{-} g=0, \quad a=1, \ldots, N,
$$

where

$$
P_{a}(r, z)=\left(\sum_{i=1}^{\infty} i v_{a i} z^{i}+q_{a} \frac{\partial}{\partial q_{a}}+\sum_{p=1}^{N} r_{p} \sum_{k \in \mathbb{Z}} E_{(k)}^{p a} z^{-k}\right) \exp \left(-\sum_{p=1}^{N} r_{p} \sum_{j=1}^{\infty} \frac{z^{-j}}{j} \frac{\partial}{\partial v_{p j}}\right) .
$$


At this point we find it convenient to make a change of variables $x_{a j}=j v_{a j}$. In this notation $P_{a}(r, z)$ takes the form

$P_{a}(r, z)=\left(\sum_{i=1}^{\infty} x_{a i} z^{i}+q_{a} \frac{\partial}{\partial q_{a}}+\sum_{p=1}^{N} r_{p} \sum_{k \in \mathbb{Z}} E_{(k)}^{p a} z^{-k}\right) \exp \left(-\sum_{p=1}^{N} r_{p} \sum_{j=1}^{\infty} z^{-j} \frac{\partial}{\partial x_{p j}}\right)$.

Let us expand $P_{a}(r, z)$ in a formal series in variables $r=\left(r_{1}, \ldots, r_{N}\right)$ :

$$
P_{a}(r, z)=\sum_{s \in \mathbb{Z}_{+}^{N}} r^{s} P_{a s}(z)
$$

It is easy to see that for any $j \in \mathbb{Z}$ and any vector $g^{\prime}$, there are only finitely many $s \in \mathbb{Z}_{+}^{N}$ such that the coefficient at $z^{j}$ in $P_{a s}(z) g^{\prime}$ is non-zero. Thus the coefficient at $z^{j}$ in $\sum_{s \in \mathbb{Z}_{+}^{N}} r^{s} P_{a s}(z) g$ is a polynomial in $r$. Since for each $j<0$ these polynomials vanish for all $r \in \mathbb{Z}^{N}$, we conclude that for all $s \in \mathbb{Z}_{+}^{N}, a=1, \ldots, N$,

$$
P_{a s}(z)_{-} g=0 \text {. }
$$

Note that for $s=0$ this equation is trivial. Let us consider the case $s \in \mathbb{Z}_{+}^{N}$, with $s_{p}=1$ and $s_{i}=0$ for $i \neq p$. This gives us the equation

$$
\begin{gathered}
\sum_{k=1}^{\infty} z^{-k} E_{(k)}^{p a} g=\left(\left(\sum_{i=1}^{\infty} x_{a i} z^{i}+q_{a} \frac{\partial}{\partial q_{a}}\right)\left(\sum_{k=1}^{\infty} z^{-k} \frac{\partial}{\partial x_{p k}}\right)\right)_{-} g \\
=\left(\sum_{k=1}^{\infty}\left(\sum_{i=1}^{k-1} x_{a i} z^{i}+q_{a} \frac{\partial}{\partial q_{a}}\right) z^{-k} \frac{\partial}{\partial x_{p k}}\right) g
\end{gathered}
$$

Substituting (9.4) into (9.3) we get

$$
P_{a}^{\prime}(r, z)_{-} g=0
$$

where

$$
\begin{aligned}
& P_{a}^{\prime}(r, z)=\left(\sum_{i=1}^{\infty} x_{a i} z^{i}+q_{a} \frac{\partial}{\partial q_{a}}+\sum_{p=1}^{N} r_{p} \sum_{k=0}^{\infty} E_{(-k)}^{p a} z^{k}\right) \exp \left(-\sum_{p=1}^{N} r_{p} \sum_{j=1}^{\infty} z^{-j} \frac{\partial}{\partial x_{p j}}\right) \\
& \quad+\exp \left(-\sum_{p=1}^{N} r_{p} \sum_{j=1}^{\infty} z^{-j} \frac{\partial}{\partial x_{p j}}\right)\left(\sum_{p=1}^{N} r_{p} \sum_{k=1}^{\infty}\left(\sum_{i=1}^{k-1} x_{a i} z^{i}+q_{a} \frac{\partial}{\partial q_{a}}\right) z^{-k} \frac{\partial}{\partial x_{p k}}\right) .
\end{aligned}
$$

Our module is $\mathbb{Z}^{N+1}$-graded via the action of operators $d_{0}, \ldots, d_{N}$, and without loss of generality we may assume that $g$ is homogeneous relative to $\mathbb{Z}^{N+1}$-grading. We will call the eigenvalue of $\beta \mathrm{Id}-d_{0}$ the degree of $g$. We use the negative sign here to make the degree non-negative. In fact the $\mathbb{Z}$-grading by degree may be defined 
on each of the tensor factors $\mathbb{C}\left[\left.x_{p j}\right|_{j=1,2, \ldots} ^{p=1, \ldots, N}\right], L_{g l_{N}}$ and $L_{V i r}$ by

$$
\begin{gathered}
\operatorname{deg}\left(x_{p j}\right)=\operatorname{deg}\left(E_{(-j)}^{a b}\right)=\operatorname{deg}\left(L_{(-j)}\right)=j, \\
\operatorname{deg}(W)=\operatorname{deg}\left(\mathrm{v}_{h}\right)=0 .
\end{gathered}
$$

On the space $\mathbb{C}\left[\left.x_{p j}\right|_{j=1,2, \ldots} ^{p=1, \ldots, N}\right]$ we will also consider a refinement of the $\mathbb{Z}$-grading by degree, where we will compute the degree in each of the $N$ families of variables. For each $a=1, \ldots, N$, define

$$
\operatorname{deg}_{a}\left(x_{p j}\right)=j \delta_{a p}
$$

Then for a monomial $y \in \mathbb{C}\left[\left.x_{p j}\right|_{j=1,2, \ldots} ^{p=1, \ldots, N}\right]$ we have

$$
\operatorname{deg}(y)=\sum_{a=1}^{N} \operatorname{deg}_{a}(y) .
$$

In addition to the degree of monomials, we will consider another $\mathbb{Z}^{N}$-grading by length, where

$$
\operatorname{len}_{a}\left(x_{p j}\right)=\delta_{a p}
$$

and define the total length to be

$$
\operatorname{len}(y)=\sum_{a=1}^{N} \operatorname{len}_{a}(y)
$$

Let us fix homogeneous bases $\left\{y_{i}^{\prime}\right\},\left\{y_{j}^{\prime \prime}\right\},\left\{y_{k}^{\prime \prime \prime}\right\}$ in the spaces $\mathbb{C}\left[\left.x_{p j}\right|_{j=1,2, \ldots} ^{p=1, \ldots, N}\right]$, $L_{g l_{N}}$ and $L_{V i r}$ respectively. Then we can expand $g$ into the finite sum

$$
g=\sum_{i j k} \alpha_{i j k} q^{\mu} y_{i}^{\prime} \otimes y_{j}^{\prime \prime} \otimes y_{k}^{\prime \prime \prime} .
$$

Note that in the above decomposition $\operatorname{deg}(g)=\operatorname{deg}\left(y_{i}^{\prime}\right)+\operatorname{deg}\left(y_{j}^{\prime \prime}\right)+\operatorname{deg}\left(y_{k}^{\prime \prime \prime}\right)$. Since equations (9.2), (9.4) and (9.5) do not involve any operators acting on the component $L_{V i r}$, we conclude that these must be satisfied not only by $g$, but also by each of the components

$$
g_{k}=\sum_{i j} \alpha_{i j k} q^{\mu} y_{i}^{\prime} \otimes y_{j}^{\prime \prime} \otimes y_{k}^{\prime \prime \prime}
$$

Lemma 9.4. Let $g$ be a homogeneous non-zero critical vector. Then in the decomposition (9.6) there exist $y_{j}^{\prime \prime} \in W$ and $y_{k}^{\prime \prime \prime} \in \mathbb{C}_{h}$ with $\alpha_{i j k} \neq 0$ for some $i$.

Proof. Let us rewrite (9.6) as

$$
g=\sum_{i j} q^{\mu} y_{i}^{\prime} \otimes y_{j}^{\prime \prime} \otimes\left(\sum_{k} \alpha_{i j k} y_{k}^{\prime \prime \prime}\right) .
$$

Consider the smallest degree $n_{0}$ of $y_{k}^{\prime \prime \prime}$ for which $\alpha_{i j k} \neq 0$ for some $i, j$. We claim that $n_{0}=0$. Otherwise, since $L_{V i r}$ is irreducible, there exists a raising operator $\omega_{(n)}^{\operatorname{Vir}}, n \geq 2$, such that

$$
\omega_{(n)}^{V i r} \sum_{k, \operatorname{deg}\left(y_{k}^{\prime \prime \prime}\right)=n_{0}} \alpha_{i j k} y_{k}^{\prime \prime \prime} \neq 0 .
$$


Now consider the Virasoro operator acting on all three factors of the tensor product: $\omega_{(n)}=\omega_{(n)}^{H y p}+\omega_{(n)}^{g l_{N}}+\omega_{(n)}^{\text {Vir }}$. The part of $\omega_{(n)} g$ involving the terms of the smallest degree in the component $L_{V i r}$ will be

$$
\sum_{i j} q^{\mu} y_{i}^{\prime} \otimes y_{j}^{\prime \prime} \otimes \omega_{(n)}^{V i r} \sum_{k, \operatorname{deg}\left(y_{k}^{\prime \prime \prime}\right)=n_{0}} \alpha_{i j k} y_{k}^{\prime \prime \prime} \neq 0 .
$$

Thus $\omega_{(n)} g \neq 0$. However, operator $\omega_{(n)}$ represents $-t_{0}^{n-1} d_{0} \in \mathcal{D}_{+}$and must annihilate $g$ since $g$ is a critical vector. This is a contradiction, which implies that $n_{0}=0$.

Let $\widetilde{g}=q^{\mu} \sum_{i j} \alpha_{i j} y_{i}^{\prime} \otimes y_{j}^{\prime \prime} \otimes \mathrm{v}_{h}$ be the projection of $g$ to the space

$$
q^{\mu} \mathbb{C}\left[\left.x_{p j}\right|_{j=1,2, \ldots} ^{p=1, \ldots, N}\right] \otimes L_{g l_{N}}(W) \otimes \mathrm{v}_{h} .
$$

We just proved that $\widetilde{g} \neq 0$, and it was noted earlier that it satisfies equation (9.4). Let $n_{1}$ be the smallest degree of $y_{j}^{\prime \prime}$ such that $\alpha_{i j} \neq 0$ for some $i$. To complete the proof of the lemma, we need to show that $n_{1}=0$. If $n_{1}>0$ using the same argument as above we see that there exists a raising operator $E_{(n)}^{p a}, n \geq 1$ such that $E_{(n)}^{p a} \widetilde{g}$ will have a non-zero component with terms in $L_{g l_{N}}(W)$ of degree $n_{1}-n$. However (9.4) implies that all factors from $L_{g l_{N}}(W)$ that appear in $E_{(n)}^{p a} \widetilde{g}$ have degrees at least $n_{1}$. This contradiction implies $n_{1}=0$, and the lemma is proved.

Let $\bar{g}$ be the projection of the critical vector $g$ to the space

$$
q^{\mu} \mathbb{C}\left[\left.x_{p j}\right|_{j=1,2, \ldots} ^{p=1, \ldots, N}\right] \otimes W \otimes \mathrm{v}_{h} .
$$

By the above lemma, $\bar{g} \neq 0$. Let us take the projection of (9.5) to the space (9.7). This yields

$$
P_{a}^{\prime \prime}(r, z)_{-} \bar{g}=0
$$

where

$$
\begin{aligned}
& P_{a}^{\prime \prime}(r, z)=\left(\sum_{i=1}^{\infty} x_{a i} z^{i}+q_{a} \frac{\partial}{\partial q_{a}}+\sum_{p=1}^{N} r_{p} E_{(0)}^{p a}\right) \exp \left(-\sum_{p=1}^{N} r_{p} \sum_{j=1}^{\infty} z^{-j} \frac{\partial}{\partial x_{p j}}\right) \\
& +\exp \left(-\sum_{p=1}^{N} r_{p} \sum_{j=1}^{\infty} z^{-j} \frac{\partial}{\partial x_{p j}}\right)\left(\sum_{p=1}^{N} r_{p} \sum_{k=1}^{\infty}\left(\sum_{i=1}^{k-1} x_{a i} z^{i}+q_{a} \frac{\partial}{\partial q_{a}}\right) z^{-k} \frac{\partial}{\partial x_{p k}}\right) .
\end{aligned}
$$

Again we decompose $P_{a}^{\prime \prime}(r, z)$ in a formal power series in $r$,

$$
P_{a}^{\prime \prime}(r, z)=\sum_{s \in \mathbb{Z}_{+}^{N}} r^{s} P_{a s}^{\prime \prime}(z),
$$

and we have $P_{a s}^{\prime \prime}(z)_{-} \bar{g}=0$ for all $s \in \mathbb{Z}_{+}^{N}$.

Next we will consider the grading of $\bar{g}$ by (total) length. Note that the operator $P_{a s}^{\prime \prime}(z)$ has two homogeneous components with respect to the length grading - one that decreases the length by $s_{1}+\ldots+s_{N}-1$ and the other (that contains terms involving $\left.q_{a} \frac{\partial}{\partial q_{a}}\right)$ by $s_{1}+\ldots+s_{N}$. Let $f$ be the maximum length component of $\bar{g}$ and suppose $\operatorname{len}(f)=\ell$. Denote by $Q_{a s}(z)$ the component of $P_{a s}^{\prime \prime}(z)$ that reduces the length by $s_{1}+\ldots+s_{N}-1$. Then the component of length $\ell+1-s_{1}-\ldots-s_{N}$ in $P_{a s}^{\prime \prime}(z)_{-} \bar{g}$ is $Q_{a s}(z)_{-} f$. Thus

$$
Q_{a s}(z)_{-} f=0
$$


Assembling back the generating series $Q_{a}(r, z)=\sum_{s \in \mathbb{Z}_{+}^{N}} r^{s} Q_{a s}(z)$, we get

$$
\begin{array}{r}
Q_{a}(r, z)=\left(\sum_{i=1}^{\infty} x_{a i} z^{i}+\sum_{p=1}^{N} r_{p} E_{(0)}^{p a}\right) \exp \left(-\sum_{p=1}^{N} r_{p} \sum_{j=1}^{\infty} z^{-j} \frac{\partial}{\partial x_{p j}}\right) \\
+\exp \left(-\sum_{p=1}^{N} r_{p} \sum_{j=1}^{\infty} z^{-j} \frac{\partial}{\partial x_{p j}}\right)\left(\sum_{p=1}^{N} r_{p} \sum_{k=1}^{\infty}\left(\sum_{i=1}^{k-1} x_{a i} z^{i}\right) z^{-k} \frac{\partial}{\partial x_{p k}}\right) \\
=\left(\sum_{i=1}^{\infty} x_{a i} z^{i}\right) \exp \left(-\sum_{p=1}^{N} r_{p} \sum_{j=1}^{\infty} z^{-j} \frac{\partial}{\partial x_{p j}}\right) \\
+\left(\sum_{p=1}^{N} r_{p} \sum_{k=1}^{\infty}\left(\sum_{i=1}^{k-1} x_{a i} z^{i}\right) z^{-k} \frac{\partial}{\partial x_{p k}}\right) \exp \left(-\sum_{p=1}^{N} r_{p} \sum_{j=1}^{\infty} z^{-j} \frac{\partial}{\partial x_{p j}}\right) \\
+\left(\sum_{p=1}^{N} r_{p} E_{(0)}^{p a}-r_{a} \sum_{k=1}^{\infty} \sum_{p=1}^{N}(k-1) r_{p} z^{-k} \frac{\partial}{\partial x_{p k}}\right) \exp \left(-\sum_{p=1}^{N} r_{p} \sum_{j=1}^{\infty} z^{-j} \frac{\partial}{\partial x_{p j}}\right) .
\end{array}
$$

Our goal is to solve the system of equations (9.9) in the space $\mathbb{C}\left[\left.x_{p j}\right|_{j=1,2, \ldots} ^{p=1, \ldots, N}\right] \otimes W$. The solutions we are looking for are homogeneous in both degree and length. Let $\operatorname{deg}(f)=m$ and $\operatorname{len}(f)=\ell$. Equation (9.9) has trivial solutions with $\ell=0$. These correspond to the critical vectors in the top of the module. We are going to show that non-trivial solutions of (9.9) must have length $\ell=1$.

To establish this claim we will first analyze (9.9) in the cases $N=1$ and $N=2$. The case of the general $N$ will follow from this simple observation. Consider a proper subset $S \subset\{1, \ldots, N\}$. Let us take a solution $f$ of $(9.9)$ and specialize all variables $x_{p j}$ with $p \notin S$ to scalars; we will get a solution for (9.9) with a smaller $N$. To see this, set in (9.9) $r_{p}=0$ for all $p \notin S$ and restrict $a$ to the set $S$. The information about the solutions of (9.9) with $N=1$ and $N=2$ may be used to establish properties of solutions of this equation for a general $N$.

Lemma 9.5. Let $N=1$, and let $W$ be a 1-dimensional gl $l_{1}$-module with identity matrix $I=E^{11}$ acting by scalar $\alpha \in \mathbb{C}$. Let $f$ be a non-constant homogeneous (in both length and degree) solution of (9.9) with $N=1$. Then $\operatorname{len}(f)=1$ and $\alpha=1-\operatorname{deg}(f)$.

Proof. First of all, let us rewrite (9.10) for the case $N=1$. To simplify notation we will drop redundant indices and write $x_{i}$ instead of $x_{p i}$, etc.:

$$
\begin{aligned}
& Q(r, z)=\left(\sum_{i=1}^{\infty} x_{i} z^{i}+r \sum_{k=1}^{\infty}\left(\sum_{i=1}^{k-1} x_{i} z^{i}\right) z^{-k} \frac{\partial}{\partial x_{k}}+r \alpha-r^{2} \sum_{k=1}^{\infty}(k-1) z^{-k} \frac{\partial}{\partial x_{k}}\right) \\
& \times \exp \left(-r \sum_{j=1}^{\infty} z^{-j} \frac{\partial}{\partial x_{j}}\right) \text {. }
\end{aligned}
$$


Let $f$ satisfy $Q(r, z)_{-} f=0$. We may view $f$ as a polynomial of length $\ell>0$ and degree $m \geq \ell$ in $\mathbb{C}\left[x_{1}, x_{2}, \ldots\right]$. Choose a natural number $s$ such that

$$
\left(\sum_{j=1}^{\infty} z^{-j} \frac{\partial}{\partial x_{j}}\right)^{s+1} f=0
$$

but

$$
R(z)=\left(\sum_{j=1}^{\infty} z^{-j} \frac{\partial}{\partial x_{j}}\right)^{s} f \neq 0
$$

Clearly $1 \leq s \leq \ell$.

Let us consider the coefficient at $r^{s+1}$ in the equation $Q(r, z)_{-} f=0$ :

$$
\begin{gathered}
\frac{(-1)^{s}}{s !}\left(\sum_{k=1}^{\infty}\left(\sum_{i=1}^{k-1} x_{i} z^{i}\right) z^{-k} \frac{\partial}{\partial x_{k}}\right) R(z)+\frac{(-1)^{s}}{s !} \alpha R(z) \\
-\frac{(-1)^{s-1}}{(s-1) !}\left(\sum_{k=1}^{\infty}(k-1) z^{-k} \frac{\partial}{\partial x_{k}}\right)\left(\sum_{j=1}^{\infty} z^{-j} \frac{\partial}{\partial x_{j}}\right)^{s-1} f=0 .
\end{gathered}
$$

Thus

$$
\left(\sum_{k=1}^{\infty}\left(\sum_{i=1}^{k-1} x_{i} z^{i}\right) z^{-k} \frac{\partial}{\partial x_{k}}\right) R(z)+\alpha R(z)-z \frac{d}{d z} R(z)-s R(z)=0 .
$$

Consider an expansion $R(z)=R_{n} z^{-n}+R_{n+1} z^{-n-1}+\ldots+R_{m} z^{-m}$ with $R_{n} \neq 0$.

Let us look at the coefficient at $z^{-n}$ in the above equation:

$$
\alpha R_{n}+n R_{n}-s R_{n}=0,
$$

which implies $\alpha=s-n \in \mathbb{Z}_{-}$.

Applying the operator $z \frac{d}{d z}$ to (9.12) we get

$$
\left(\sum_{k=1}^{\infty} k z^{-k} \frac{\partial}{\partial x_{k}}\right) R(z)=0 .
$$

Let us prove by induction that for all $j \geq 0$,

$$
\left(\sum_{k=1}^{\infty} k^{j} z^{-k} \frac{\partial}{\partial x_{k}}\right) R(z)=0
$$

Suppose (9.15) holds for all $j^{\prime} \leq j, j \geq 1$. Applying the operator $\sum_{k=1}^{\infty} k^{j} z^{-k} \frac{\partial}{\partial x_{k}}$ to (9.14) and using the induction assumption, we get

$$
\begin{gathered}
0=\left(\sum_{p=1}^{\infty} p^{j} z^{-p} \frac{\partial}{\partial x_{p}}\right)\left(\sum_{k=1}^{\infty}\left(\sum_{i=1}^{k-1} x_{i} z^{i}\right) z^{-k} \frac{\partial}{\partial x_{k}}\right) R(z)-\left(\sum_{k=1}^{\infty} k^{j} z^{-k} \frac{\partial}{\partial x_{k}}\right) z \frac{d}{d z} R(z) \\
=\left(\sum_{k=1}^{\infty}\left(\sum_{p=1}^{k-1} p^{j}\right) z^{-k} \frac{\partial}{\partial x_{k}}\right) R(z)-\left(\sum_{k=1}^{\infty} k^{j+1} z^{-k} \frac{\partial}{\partial x_{k}}\right) R(z) \\
=\left(\frac{1}{j+1}-1\right)\left(\sum_{k=1}^{\infty} k^{j+1} z^{-k} \frac{\partial}{\partial x_{k}}\right) R(z),
\end{gathered}
$$


which establishes the inductive step. In the above calculation we used the fact that $\sum_{p=1}^{k-1} p^{j}$ is a polynomial in $k$ with the leading term $\frac{k^{j+1}}{j+1}$.

Using the Vandermonde determinant argument we conclude from (9.15) that

$$
\frac{\partial}{\partial x_{k}} R(z)=0
$$

for all $k$. This implies that all $R_{i}$ are scalars, but since they can't have equal degrees, we conclude that $R_{n}$ is a non-zero scalar, while all other coefficients are zero. Without the loss of generality we may thus assume

$$
R(z)=\left(\sum_{j=1}^{\infty} z^{-j} \frac{\partial}{\partial x_{j}}\right)^{s} f=z^{-n} .
$$

Thus $s=\operatorname{len}(f)=\ell$ and $n=\operatorname{deg}(f)=m$, and then $\alpha=\operatorname{len}(f)-\operatorname{deg}(f)$. It remains to prove that $\operatorname{len}(f)=1$. We will reason by contradiction. Let us suppose that $\ell=\operatorname{len}(f)>1$ and consider

$$
S(z)=\left(\sum_{j=1}^{\infty} z^{-j} \frac{\partial}{\partial x_{j}}\right)^{\ell-1} f
$$

Since every term in $S(z)$ has length 1 , we can write

$$
S(z)=\beta_{1} x_{1} z^{-m+1}+\beta_{2} x_{2} z^{-m+2}+\ldots+\beta_{m-\ell+1} x_{m-\ell+1} z^{-\ell+1} .
$$

Now we look at the coefficient at $r^{\ell}$ in the equation $Q(r, z)_{-} f=0$ :

$$
\begin{gathered}
\left.0=\frac{(-1)^{\ell}}{\ell !}\left(\left(\sum_{i=1}^{\infty} x_{i} z^{i}\right)\left(\sum_{j=1}^{\infty} z^{-j} \frac{\partial}{\partial x_{j}}\right)^{\ell} f\right)_{-}\right)^{\ell-1} \\
+\frac{(-1)^{\ell-1}}{(\ell-1) !}\left(\sum_{k=1}^{\infty}\left(\sum_{i=1}^{k-1} x_{i} z^{i}\right) z^{-k} \frac{\partial}{\partial x_{k}}\right)\left(\sum_{j=1}^{\infty} z^{-j} \frac{\partial}{\partial x_{j}}\right)^{\ell-1} f \\
-\frac{(-1)^{\ell-2}}{(\ell-2) !}\left(\sum_{k=1}^{\infty}(k-1) z^{-k} \frac{\partial}{\partial x_{k}}\right)\left(\sum_{j=1}^{\infty} z^{-j} \frac{\partial}{\partial x_{j}}\right)^{\ell-2} f \\
+\frac{(-1)^{\ell-1}}{(\ell-1) !} \alpha\left(\sum_{j=1}^{\infty} z^{-j} \frac{\partial}{\partial x_{j}}\right)^{\ell-1} f .
\end{gathered}
$$


Taking out the factor of $\frac{(-1)^{\ell}}{\ell !}$ we get

$$
\begin{gathered}
0=\sum_{i=1}^{m-1} x_{i} z^{-m+i}-\ell\left(\sum_{k=1}^{\infty}\left(\sum_{i=1}^{k-1} x_{i} z^{i}\right) z^{-k} \frac{\partial}{\partial x_{k}}\right) S(z) \\
+\ell z \frac{d}{d z} S(z)+\ell(\ell-1) S(z)+\ell(m-\ell) S(z) \\
=\sum_{i=1}^{m-1} x_{i} z^{-m+i}-\ell \sum_{k=1}^{m-\ell+1}\left(\sum_{i=1}^{k-1} x_{i} z^{i}\right) \beta_{k} z^{-m} \\
+\ell \sum_{k=1}^{m-\ell+1}(-m+k) \beta_{k} x_{k} z^{-m+k}+\ell(m-1) \sum_{k=1}^{m-\ell+1} \beta_{k} x_{k} z^{-m+k} \\
=\sum_{i=1}^{m-1} x_{i} z^{-m+i}-\ell \sum_{i=1}^{m-\ell}\left(\sum_{k=i+1}^{m-\ell+1} \beta_{k}\right) x_{i} z^{-m+i}+\ell \sum_{k=1}^{m-\ell+1}(k-1) \beta_{k} x_{k} z^{-m+k} .
\end{gathered}
$$

We stress that the above calculation is only valid when $\ell>1$. If $\ell>2$ we immediately get a contradiction since the coefficient at $z^{-1}$ yields $x_{m-1}=0$. It only remains to rule out the case $\ell=2$. In the latter case the equation simplifies to the following:

$$
\sum_{k=1}^{m-1} x_{k} z^{-m+k}-2 \sum_{k=1}^{m-1}\left(\sum_{i=1}^{k-1} x_{i} z^{i}\right) \beta_{k} z^{-m}+2 \sum_{k=1}^{m-1}(k-1) \beta_{k} x_{k} z^{-m+k}=0 .
$$

Let us specialize this equation to $x_{1}=\ldots=x_{m-1}=1, z=1$. Then we get

$$
(m-1)-2 \sum_{k=1}^{m-1}(k-1) \beta_{k}+2 \sum_{k=1}^{m-1}(k-1) \beta_{k}=0
$$

which implies $m=1$, which is impossible since $m=\operatorname{deg}(f)$ cannot be less than $\ell=\operatorname{len}(f)$. Thus $\ell=\operatorname{len}(f)=1$ and the lemma is proved.

Next we are going to show that for a general $N$, a homogeneous non-trivial solution of (9.9) must have total length 1 . The previous lemma implies that such a solution may have only two components with respect to $\operatorname{len}_{a}$ grading for each $a$, where $\operatorname{len}_{a}$ may be either 0 or 1 . To prove the general case, it is sufficient to consider $N=2$, since if a monomial has $\operatorname{len}_{a}+\operatorname{len}_{b}$ at most 1 for any pair of distinct indices, then its total length does not exceed 1.

Lemma 9.6. Let $N=2$ and let $W$ be a finite-dimensional $g l_{2}$-module. Then any homogeneous (in both length and degree) non-constant solution $f$ of (9.9) has total length 1.

Proof. It is sufficient to consider the case of $W$ being irreducible since (9.9) is compatible with the $g l_{N}$-module homomorphisms. Let us fix a basis $\left\{w_{n}, w_{n-1}, \ldots, w_{-n}\right\}$ of $W$, where $n \in \frac{1}{2} \mathbb{Z}_{+}$and $\left(E^{11}-E^{22}\right) w_{i}=2 i w_{i}$. Assuming that the identity matrix acts on $W$ by scalar $\alpha$, we get

$$
E^{11} w_{i}=\left(\frac{\alpha}{2}+i\right) w_{i} \text { and } E^{22} w_{i}=\left(\frac{\alpha}{2}-i\right) w_{i}
$$

It follows from the previous lemma that every monomial in the decomposition of $f$ has length at most 1 with respect to each of the two indices. Thus we only need to prove that $f$ cannot have total length 2 . We will reason by contradiction. 
If len $(f)=2$, then for each monomial in $f$ both $\operatorname{len}_{1}$ and $\operatorname{len}_{2}$ are 1 . Suppose $\operatorname{deg}(f)=m$. Let us write

$$
f=\sum_{i=-n}^{n} f_{i} \otimes w_{i}
$$

By Lemma 9.5 we have

$$
\operatorname{deg}_{1}\left(f_{i}\right)=1-\left(\frac{\alpha}{2}+i\right), \quad \operatorname{deg}_{2}\left(f_{i}\right)=1-\left(\frac{\alpha}{2}-i\right),
$$

so $m=\operatorname{deg}(f)=2-\alpha$. Let $b_{i}=\operatorname{deg}_{1}\left(f_{i}\right)=\frac{m}{2}-i$ and $c_{i}=\operatorname{deg}_{2}\left(f_{i}\right)=\frac{m}{2}+i$. Then $f$ may be written as

$$
f=\sum_{i=-n}^{n} \beta_{i} x_{1, b_{i}} x_{2, c_{i}} \otimes w_{i}
$$

where we set $\beta_{i}=0$ whenever $b_{i} \leq 0$ or $c_{i} \leq 0$.

Let us take the equation derived from (9.9) by taking the coefficient at $r_{1} r_{2}$ with $a=1$ and substitute (9.16) in it. We get

$$
\begin{aligned}
0 & =\sum_{i=-n}^{n} \beta_{i}\left(\sum_{j=1}^{m-1} z^{-m+j} x_{1, j}\right) \otimes w_{i}-\sum_{i=-n}^{n} \beta_{i}\left(\sum_{j=1}^{b_{i}-1} z^{-m+j} x_{1, j}\right) \otimes w_{i} \\
& -\sum_{i=-n}^{n} \beta_{i}\left(\sum_{j=1}^{c_{i}-1} z^{-m+j} x_{1, j}\right) \otimes w_{i}-\sum_{i=-n}^{n} \beta_{i}\left(c_{i}-1\right) z^{-m+b_{i}} x_{1, b_{i}} \otimes w_{i} \\
& -\sum_{i=-n}^{n} \beta_{i}\left(1-b_{i}\right) z^{-m+b_{i}} x_{1, b_{i}} \otimes w_{i}-\sum_{i=-n}^{n} \beta_{i} z^{-m+c_{i}} x_{2, c_{i}} \otimes E^{21} w_{i} .
\end{aligned}
$$

Note that only the last sum contains variables $x_{2, j}$. By equating this sum to zero, we conclude that $\beta_{i} E^{21} w_{i}=0$ for all $i=-n, \ldots, n$. It follows that $\beta_{i}=0$ for all $i \neq-n$. Similarly, taking the same equation with $a=2$, we will get that $\beta_{i}=0$ for all $i \neq n$. Thus the only possibility for a non-zero solution is when $n=0$, which means that $W$ is 1-dimensional and $m$ is even. In this case $b_{0}=c_{0}=\frac{m}{2}$ and $f=x_{1, \frac{m}{2}} x_{2, \frac{m}{2}} \otimes w$, and (9.17) becomes

$$
\sum_{j=1}^{m-1} x_{1, j} z^{-m+j} \otimes w-2 \sum_{j=1}^{\frac{m}{2}-1} z^{-m+j} x_{1, j} \otimes w=0,
$$

which gives a contradiction. Thus the total length of $f$ must be 1 and the lemma is proved.

Now we return to the general case. We proved that a non-trivial homogeneous solution $f$ of $(9.9)$ must have length 1 . Suppose $\operatorname{deg}(f)=m$. Then $f$ can be written as

$$
f=\sum_{p=1}^{N} x_{p m} \otimes w_{p}, \quad w_{p} \in W
$$


Equation (9.9) then simplifies as follows:

$$
\begin{aligned}
0 & =\sum_{p=1}^{N} r_{p} \sum_{b=1}^{N} r_{b} E^{p a} w_{b}+r_{a}(m-1) \sum_{b=1}^{N} r_{b} w_{b} \\
& =\sum_{p=1}^{N} \sum_{b=1}^{N} r_{p} r_{b}\left(E^{p a}+\delta_{p a}(m-1)\right) w_{b} .
\end{aligned}
$$

Consider a new action $\rho^{\prime}$ of $g l_{N}$ on $W$ :

$$
\rho^{\prime}\left(E^{p a}\right) w=E^{p a} w+(m-1) \delta_{a p} w, \quad w \in W .
$$

This gives the same structure of $W$ as an $s l_{N}$-module, but now the identity matrix acts with scalar $\alpha^{\prime}=\alpha+(m-1) N$. Then (9.18) is equivalent to the system of equations

$$
\rho^{\prime}\left(E^{c a}\right) w_{b}+\rho^{\prime}\left(E^{b a}\right) w_{c}=0
$$

where $a, b, c=1, \ldots, N$.

We will also use a third $g l_{N}$-action $\rho^{\prime \prime}$ on $W$ :

$$
\rho^{\prime \prime}\left(E^{p a}\right) w=\rho^{\prime}\left(E^{p a}\right) w+\delta_{a p} w=E^{p a} w+m \delta_{a p} w, \quad w \in W .
$$

The identity matrix here acts with scalar $\alpha^{\prime \prime}=\alpha+m N$. For this action (9.19) may be written as

$$
\rho^{\prime \prime}\left(E^{c a}\right) w_{b}+\rho^{\prime \prime}\left(E^{b a}\right) w_{c}=\delta_{c a} w_{b}+\delta_{b a} w_{c}
$$

We also have

$$
\sum_{p=1}^{N} \sum_{b=1}^{N} r_{p} r_{b} \rho^{\prime \prime}\left(E^{p a}\right) w_{b}=r_{a} \sum_{b=1}^{N} r_{b} w_{b} .
$$

We are going to classify $g l_{N}$-modules $W$ for which the system (9.19) has nontrivial solutions. We will do this indirectly, linking this system with reducibility of tensor modules.

Lemma 9.7. Let $\left(W, \rho^{\prime \prime}\right)$ be a finite-dimensional irreducible $g l_{N}$-module. Let $\mathcal{P}$ be the set of all solutions $\left(w_{1}, \ldots, w_{N}\right) \in W \times \ldots \times W$ of the system of equations (9.20). Then the subspace

$$
\widetilde{\mathcal{P}}=\left\{\underset{r \in \mathbb{Z}^{N}}{\oplus} q^{r} \otimes\left(r_{1} w_{1}+\ldots+r_{N} w_{N}\right) \mid\left(w_{1}, \ldots, w_{N}\right) \in \mathcal{P}\right\}
$$

is a Vect $\mathbb{T}^{N}$-submodule in the tensor module $T(W)=\mathbb{C}\left[q_{1}^{ \pm 1}, \ldots, q_{N}^{ \pm 1}\right] \otimes W$, associated with $\left(W, \rho^{\prime \prime}\right)$.

Proof. Let $\left(w_{1}, \ldots, w_{N}\right) \in \mathcal{P}$. Then using the tensor module action and (9.21) we get

$$
\begin{gathered}
t^{s} d_{a}\left(q^{r} \otimes \sum_{b=1}^{N} r_{b} w_{b}\right)=r_{a} q^{r+s} \otimes \sum_{b=1}^{N} r_{b} w_{b}+\sum_{p=1}^{N} \sum_{b=1}^{N} s_{p} r_{b} q^{r+s} \otimes \rho^{\prime \prime}\left(E^{p a}\right) w_{b} \\
=\sum_{b=1}^{N} r_{b} q^{r+s} \otimes \sum_{p=1}^{N}\left(r_{p}+s_{p}\right) \rho^{\prime \prime}\left(E^{p a}\right) w_{b} .
\end{gathered}
$$

Fix $1 \leq a, i \leq N$. Set $\widetilde{w}_{p}=\rho^{\prime \prime}\left(E^{p a}\right) w_{i}, p=1, \ldots, N$. To complete the proof of the lemma, it is sufficient to show that $\left(\widetilde{w}_{1}, \ldots, \widetilde{w}_{N}\right) \in \mathcal{P}$. Instead of working 
with (9.20), it will be easier to check the equivalent condition (9.19). Note that $\widetilde{w}_{p}=\rho^{\prime}\left(E^{p a}\right) w_{i}+\delta_{p a} w_{i}$. Then using the fact that $\left(w_{1}, \ldots, w_{N}\right)$ satisfies $(9.19)$, we obtain

$$
\begin{gathered}
\rho^{\prime}\left(E^{c d}\right) \widetilde{w}_{b}+\rho^{\prime}\left(E^{b d}\right) \widetilde{w}_{c} \\
=\rho^{\prime}\left(E^{c d}\right) \rho^{\prime}\left(E^{b a}\right) w_{i}+\delta_{a b} \rho^{\prime}\left(E^{c d}\right) w_{i}+\rho^{\prime}\left(E^{b d}\right) \rho^{\prime}\left(E^{c a}\right) w_{i}+\delta_{a c} \rho^{\prime}\left(E^{b d}\right) w_{i} \\
=-\rho^{\prime}\left(E^{c d}\right) \rho^{\prime}\left(E^{i a}\right) w_{b}+\delta_{a b} \rho^{\prime}\left(E^{c d}\right) w_{i}-\rho^{\prime}\left(E^{b d}\right) \rho^{\prime}\left(E^{i a}\right) w_{c}+\delta_{a c} \rho^{\prime}\left(E^{b d}\right) w_{i} \\
=-\rho^{\prime}\left(E^{i a}\right) \rho^{\prime}\left(E^{c d}\right) w_{b}-\delta_{i d} \rho^{\prime}\left(E^{c a}\right) w_{b}+\delta_{a c} \rho^{\prime}\left(E^{i d}\right) w_{b}+\delta_{a b} \rho^{\prime}\left(E^{c d}\right) w_{i} \\
-\rho^{\prime}\left(E^{i a}\right) \rho^{\prime}\left(E^{b d}\right) w_{c}-\delta_{i d} \rho^{\prime}\left(E^{b a}\right) w_{c}+\delta_{a b} \rho^{\prime}\left(E^{i d}\right) w_{c}+\delta_{a c} \rho^{\prime}\left(E^{b d}\right) w_{i}=0 .
\end{gathered}
$$

Thus $\left(\widetilde{w}_{1}, \ldots, \widetilde{w}_{N}\right) \in \mathcal{P}$. The lemma is now proved.

Corollary 9.8. Let $(W, \rho)$ be a finite-dimensional irreducible $g l_{N}$-module. If $L(W, \gamma, h)$ has a critical vector of degree $m \geq 1$, then either $W$ has a fundamental highest weight $\omega_{k}, 1 \leq k \leq N-1$, with respect to sl ${ }_{N}$-action, with identity matrix acting with scalar $\alpha=k-m N$, or $W$ is a 1-dimensional module with identity matrix acting with scalar $\alpha=N-m N$.

Proof. If the system (9.20) has a non-trivial solution, then the submodule $\widetilde{\mathcal{P}}$ in the tensor module $T(W)$ corresponding to $\left(W, \rho^{\prime \prime}\right)$ is non-zero. It is a proper submodule since its component at $q^{0}$ is trivial. Using the classification of reducible tensor modules (Theorem 2.1), we conclude that $T(W)$ is one of the de Rham modules $\Omega^{k}\left(\mathbb{T}^{N}\right), k=1, \ldots, N$. Taking into account the relation $\alpha=\alpha^{\prime \prime}-m N$, we obtain the claim of the corollary.

To complete the proof of Theorem 9.1 it remains to establish the following.

Lemma 9.9. If $L(W, \gamma, h)$ has a critical vector that does not belong on the top, then $h=0$.

Proof. The statement can be proved using the same method which was used in the derivation of (9.9). We will only sketch the idea of the proof here. A critical vector $g$ is annihilated by $t_{0}^{j} t^{r} d_{0}$ for $j \geq 0$. Thus

$$
\left(z^{2} d_{0}(r, z)\right)_{-} g=0 .
$$

Using (6.12) we can rewrite that equation explicitly and then project everything to the subspace (9.7), which will give a system of equations on $f$. More precisely, one should look at the $r_{a}$-component in that system. Since we have by now a fairly detailed description of $f$, the resulting equation can be simplified yielding $h=0$.

\section{Chiral de Rham complex}

The chiral de Rham complex was introduced by Malikov et al. in [20]. In the case a of torus $\mathbb{T}^{N}$, the space of this differential complex is a tensor product of two vertex (super) algebras

$$
V_{H y p}^{+} \otimes V_{\mathbb{Z}^{N}}
$$

More generally one can use a shift by $\gamma$ and consider instead the space $M_{H y p}(\gamma) \otimes$ $V_{\mathbb{Z}^{N}}$. Here $V_{\mathbb{Z}^{N}}$ is the lattice vertex superalgebra of the standard euclidean lattice $\mathbb{Z}^{N}$. Before we define the differential of this complex, let us review the structure of $V_{\mathbb{Z}^{N}}$. The vertex superalgebra $V_{\mathbb{Z}^{N}}$ has two main realizations - the bosonic realization and the fermionic one, with boson-fermion correspondence being an 
isomorphism between the two models. For our purposes it will be more convenient to use the fermionic realization of $V_{\mathbb{Z}^{N}}$.

Consider the Clifford Lie superalgebra $C l_{N}$ of "charged free fermions" with basis

$$
\left\{\varphi_{(j)}^{p}, \psi_{(j)}^{p} \mid p=1, \ldots, N, j \in \mathbb{Z}\right\}
$$

of its odd part and a 1-dimensional even part spanned by a central element $K$. The Lie bracket in $C l_{N}$ is given by

$$
\left[\varphi_{(m)}^{a}, \psi_{(n)}^{b}\right]=\delta_{a b} \delta_{m,-n-1} K, \quad\left[\varphi_{(m)}^{a}, \varphi_{(n)}^{b}\right]=\left[\psi_{(m)}^{a}, \psi_{(n)}^{b}\right]=0 .
$$

Define formal fields

$$
\varphi^{a}(z)=\sum_{j \in \mathbb{Z}} \varphi_{(j)}^{a} z^{-j-1}, \quad \psi^{a}(z)=\sum_{j \in \mathbb{Z}} \psi_{(j)}^{a} z^{-j-1}, \quad K(z)=K z^{0} .
$$

With this choice of fields $C l_{N}$ becomes a vertex Lie superalgebra since the only non-trivial relation between these fields is

$$
\left[\varphi^{a}\left(z_{1}\right), \psi^{b}\left(z_{2}\right)\right]=\delta_{a b} K\left(z_{2}\right)\left[z_{1}^{-1} \delta\left(\frac{z_{2}}{z_{1}}\right)\right] .
$$

The lattice vertex superalgebra $V_{\mathbb{Z}^{N}}$ is isomorphic to the universal enveloping vertex algebra of $C l_{N}$ at level 1 . As a vector space it is the unique $C l_{N}$-module generated by vacuum vector $\mathbb{1}$ satisfying

$$
K \mathbb{1}=\mathbb{1}, \quad \varphi_{(j)}^{p} \mathbb{1}=\psi_{(j)}^{p} \mathbb{1}=0 \text { for } j \geq 0, p=1, \ldots, N .
$$

In its fermionic realization $V_{\mathbb{Z}^{N}}$ is the exterior algebra with generators $\left\{\varphi_{(j)}^{p}\right.$, $\left.\left.\psi_{(j)}^{p}\right|_{j \leq-1} ^{p=1, \ldots, N}\right\}$ and is irreducible as a module over $C l_{N}$. The state-field correspondence map $Y$ is given by the standard formula (6.1).

We fix the Virasoro element in $V_{\mathbb{Z}^{N}}$ :

$$
\omega^{f e r}=\sum_{p=1}^{N} \varphi_{(-2)}^{p} \psi_{(-1)}^{p} \mathbb{1} .
$$

The rank of this VOA is $-2 N$.

It is well known that vertex superalgebra $V_{\mathbb{Z}^{N}}$ contains a level 1 simple $\widehat{g l}_{N}$ vertex algebra. The fields generating this subalgebra are

$$
E^{a b}(z)=: \varphi^{a}(z) \psi^{b}(z): .
$$

It is easy to check that these fields satisfy relations (6.4) and the central element of $\widehat{g l}_{N}$ acts as the identity operator. It is also straightforward to verify that the Virasoro element (6.7) in the $\widehat{g l}_{N}$ vertex algebra maps to $\omega^{f e r}$ under this embedding.

Let us define two $\mathbb{Z}$-gradings on $V_{\mathbb{Z}^{N}}$. The fermionic degree is defined by

$$
\operatorname{deg}_{f e r}\left(\varphi_{(j)}^{p}\right)=1, \quad \operatorname{deg}_{f e r}\left(\psi_{(j)}^{p}\right)=-1, \quad \operatorname{deg}_{f e r}(K)=\operatorname{deg}_{f e r}(\mathbb{1})=0 .
$$

The bosonic grading is defined as follows:

$$
\operatorname{deg}_{b o s}\left(\varphi_{(j)}^{p}\right)=-j-1, \quad \operatorname{deg}_{b o s}\left(\psi_{(j)}^{p}\right)=-j, \quad \operatorname{deg}_{b o s}(K)=\operatorname{deg}_{b o s}(\mathbb{1})=0 .
$$

Let $V_{\mathbb{Z}^{N}}^{k}$ be the subspace of the elements of fermionic degree $k$. We have a decomposition

$$
V_{\mathbb{Z}^{N}}=\bigoplus_{k \in \mathbb{Z}} V_{\mathbb{Z}^{N}}^{k}
$$


Note that each subspace $V_{\mathbb{Z}^{N}}^{k}$ is a $\widehat{g l}_{N}$-submodule which is graded by the bosonic degree. Its structure is described by the following well-known result (see e.g. [9] or [15]):

Theorem 10.1. For each $k \in \mathbb{Z}, V_{\mathbb{Z}^{N}}^{k}$ is an irreducible $\widehat{g l}_{N}$-module at level 1 . Let $\widetilde{V}_{\mathbb{Z}^{N}}^{k}$ be the non-trivial component of $V_{\mathbb{Z}^{N}}^{k}$ of lowest bosonic degree. If $k=0$ $\bmod N$, then $\widetilde{V}_{\mathbb{Z}^{N}}^{k}$ is 1-dimensional. If $k=k^{\prime} \bmod N$ with $1 \leq k^{\prime}<N$, then as an sl ${ }_{N}$-module $\widetilde{V}_{\mathbb{Z}^{N}}^{k}$ has the fundamental highest weight $\omega_{k^{\prime}}$. The identity matrix of $g l_{N}$ acts on $\widetilde{V}_{\mathbb{Z}^{N}}^{k}$ as $k \mathrm{Id}$.

Combining this result with Theorem 6.3 we get

Corollary 10.2. The space

$$
M_{H y p}(\gamma) \otimes V_{\mathbb{Z}^{N}}^{k}
$$

has a structure of a module for the Lie algebra of vector fields Vect $\mathbb{T}^{N+1}$.

For these modules the Virasoro tensor factor $L_{V i r}(h)$ is 1-dimensional $(h=0)$. The modules in this family are precisely the exceptional modules $L(W, \gamma, h)$ for which Theorem 9.2 does not claim irreducibility. We are going to see below that these modules are in fact reducible.

Let us express the action $(6.11),(6.12)$ of the Lie algebra $\operatorname{Vect} \mathbb{T}^{N+1}$ on $M_{H y p}(\gamma) \otimes$ $V_{\mathbb{Z}^{N}}^{k}$ using the fermionic realization

$$
d_{a}(r, z) \mapsto Y\left(d_{a}(r), z\right), \quad d_{0}(r, z) \mapsto Y\left(d_{0}(r), z\right),
$$

where

$$
\begin{gathered}
d_{a}(r)=v_{(-1)}^{a} q^{r}+\sum_{p=1}^{N} r_{p} \varphi_{(-1)}^{p} \psi_{(-1)}^{a} q^{r} \\
d_{0}(r)=-\left(\omega_{(-1)}^{H y p} q^{r}+\omega_{(-1)}^{f e r} q^{r}+\sum_{a, b=1}^{N} r_{a} u_{(-1)}^{b} \varphi_{(-1)}^{a} \psi_{(-1)}^{b} q^{r}-\sum_{p=1}^{N} r_{p} u_{(-2)}^{p} q^{r}\right) \\
=-\left(\sum_{p=1}^{N} u_{(-1)}^{p} v_{(-1)}^{p} q^{r}+\sum_{p=1}^{N} \varphi_{(-2)}^{p} \psi_{(-1)}^{p} q^{r}+\sum_{a, b=1}^{N} r_{a} u_{(-1)}^{b} \varphi_{(-1)}^{a} \psi_{(-1)}^{b} q^{r}\right)
\end{gathered}
$$

Here we used the relation

$$
\omega_{(-1)}^{H y p} q^{r}=\sum_{p=1}^{N} u_{(-1)}^{p} v_{(-1)}^{p} q^{r}+\sum_{p=1}^{N} r_{p} u_{(-2)}^{p} q^{r} .
$$

Following 20, let us now introduce the differential

$$
\ldots \stackrel{\mathbf{d}}{\longrightarrow} M_{H y p}(\gamma) \otimes V_{\mathbb{Z}^{N}}^{k} \stackrel{\mathbf{d}}{\longrightarrow} M_{H y p}(\gamma) \otimes V_{\mathbb{Z}^{N}}^{k+1} \stackrel{\mathbf{d}}{\longrightarrow} \ldots
$$

of the chiral de Rham complex. 
Let

$$
Q=\sum_{p=1}^{N} v_{(-1)}^{p} \varphi_{(-1)}^{p} \mathbb{1}
$$

and set $\mathbf{d}=Q_{(0)}$, i.e., $\mathbf{d}$ is the coefficient at $z^{-1}$ in $Y(Q, z)=\sum_{p=1}^{N} v^{p}(z) \varphi^{p}(z)$. Vanishing of the supercommutator

$$
\left[Y\left(Q, z_{1}\right), Y\left(Q, z_{2}\right)\right]=0
$$

implies $\mathbf{d} \circ \mathbf{d}=0$ (see e.g. Lemma 10.4 below).

Theorem 10.3. The map

$$
\mathbf{d}: M_{H y p}(\gamma) \otimes V_{\mathbb{Z}^{N}}^{k} \rightarrow M_{H y p}(\gamma) \otimes V_{\mathbb{Z}^{N}}^{k+1}
$$

is a homomorphism of $\operatorname{Vect} \mathrm{T}^{N+1}$-modules.

The statement of this theorem is equivalent to the claim that the following operators on $M_{H y p}(\gamma) \otimes V_{\mathbb{Z}^{N}}$ commute:

$$
\begin{aligned}
& {\left[\mathbf{d}, d_{a}(r, z)\right]=0,} \\
& {\left[\mathbf{d}, d_{0}(r, z)\right]=0 .}
\end{aligned}
$$

The proof of these relations will be based on the following simple observation:

Lemma 10.4. Let $V$ be a vertex superalgebra and let $a, b \in V$. Suppose that $a_{(0)} b=0$. Then

$$
\left[a_{(0)}, Y(b, z)\right]=0 \text {. }
$$

Proof. Since $a_{(0)} b=0$, the commutator formula (5.2) yields

$$
\left[Y\left(a, z_{1}\right), Y\left(b, z_{2}\right)\right]=\sum_{j \geq 1} \frac{1}{j !} Y\left(a_{(j)} b, z_{2}\right)\left[z_{1}^{-1}\left(\frac{\partial}{\partial z_{2}}\right)^{j} \delta\left(\frac{z_{2}}{z_{1}}\right)\right]
$$

However the right hand side does not contain terms with $z_{1}^{-1}$, and the claim of the lemma follows.

Let us continue with the proof of the theorem. We need to show that

$$
Q_{(0)} d_{a}(r)=0 \quad \text { and } \quad Q_{(0)} d_{0}(r)=0 .
$$

Since $Y(Q, z)=\sum_{i=1}^{N}: v^{i}(z) \varphi^{i}(z):$, we have

$$
Q_{(0)}=\sum_{i=1}^{N}\left(\sum_{j=0}^{\infty} \varphi_{(-j-1)}^{i} v_{(j)}^{i}+\sum_{j=1}^{\infty} v_{(-j)}^{i} \varphi_{(j-1)}^{i}\right) .
$$


It is easy to see that $v_{(j)}^{i} d_{a}(r)=0$ for $j \geq 1$ and $\varphi_{(j)}^{i} d_{a}(r)=0$ for $j \geq 1$. Thus

$$
\begin{gathered}
Q_{(0)} d_{a}(r)=\sum_{i=1}^{N}\left(\varphi_{(-1)}^{i} v_{(0)}^{i}+v_{(-1)}^{i} \varphi_{(0)}^{i}\right) d_{a}(r) \\
=\sum_{i=1}^{N} r_{i} \varphi_{(-1)}^{i} v_{(-1)}^{a} q^{r}-\sum_{i=1}^{N} \sum_{p=1}^{N} r_{p} v_{(-1)}^{i} \varphi_{(-1)}^{p} \varphi_{(0)}^{i} \psi_{(-1)}^{a} q^{r} \\
=\sum_{i=1}^{N} r_{i} \varphi_{(-1)}^{i} v_{(-1)}^{a} q^{r}-\sum_{p=1}^{N} r_{p} v_{(-1)}^{a} \varphi_{(-1)}^{p} q^{r}=0 .
\end{gathered}
$$

The calculation for $Q_{(0)} d_{0}(r)$ is analogous, and we omit it. This completes the proof of the theorem.

Let us present a diagram of the chiral de Rham complex for $N=2$. In the diagram, the fermionic degree increases in the horizontal direction and bosonic in the vertical.

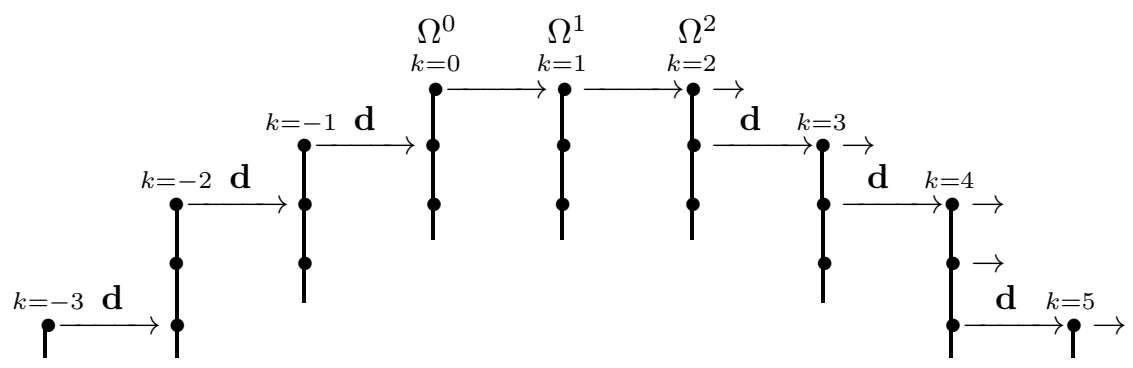

The tops of the modules $M_{H y p}(\gamma) \otimes V_{\mathbb{Z}^{N}}^{k}$ with $0 \leq k \leq N$ are the spaces $q^{\gamma} \Omega^{k}\left(\mathbb{T}^{N}\right)$ of differential $k$-forms that form the classical de Rham complex. Nontrivial Vect $\mathbb{T}^{N}$-submodules in these tops generate non-trivial Vect $\mathbb{T}^{N+1}$ submodules in corresponding modules $M_{H y p}(\gamma) \otimes V_{\mathbb{Z}^{N}}^{k}$.

It was proved in 20] that the cohomology of the chiral de Rham complex coincides with the classical de Rham cohomology. This implies, in particular, that for $k<0$ or $k>N$ the short sequences

$$
M_{H y p}(\gamma) \otimes V_{\mathbb{Z}^{N}}^{k-1} \stackrel{\mathbf{d}}{\longrightarrow} M_{H y p}(\gamma) \otimes V_{\mathbb{Z}^{N}}^{k} \stackrel{\mathbf{d}}{\longrightarrow} M_{H y p}(\gamma) \otimes V_{\mathbb{Z}^{N}}^{k+1}
$$

are exact. Using this fact, we get

Corollary 10.5. (i) For $k \leq 0$, Vect $\mathbb{T}^{N+1}$-modules $M_{H y p}(\gamma) \otimes V_{\mathbb{Z}^{N}}^{k}$ have non-trivial critical vectors.

(ii) For $k \geq N$, Vect $\mathbb{T}^{N+1}$-modules $M_{H y p}(\gamma) \otimes V_{\mathbb{Z}^{N}}^{k}$ are not generated by their top spaces.

Proof. We can see from the above diagram that for $k<0$ the images of the top vectors in $M_{H y p}(\gamma) \otimes V_{\mathbb{Z}^{N}}^{k}$ are non-trivial critical vectors in $M_{H y p}(\gamma) \otimes V_{\mathbb{Z}^{N}}^{k+1}$. For $k \geq N$, the top spaces of $M_{H y p}(\gamma) \otimes V_{\mathbb{Z}^{N}}^{k}$ are in the kernel of $\mathbf{d}$. Thus the submodules generated by the tops are annihilated by $\mathbf{d}$ as well. Since the map $\mathbf{d}$ is non-zero, these submodules are proper. 
As a result we see that all modules that belong to the chiral de Rham complex are reducible. The claim of Corollary 10.5 is consistent with the existence of the contragredient pairing given by Theorem 8.2

$$
\left(M_{H y p}(\gamma) \otimes V_{\mathbb{Z}^{N}}^{k}\right) \times\left(M_{H y p}(\gamma) \otimes V_{\mathbb{Z}^{N}}^{N-k}\right) \rightarrow \mathbb{C} .
$$

For the chiral de Rham complex this duality was constructed in [19].

\section{ACKNOWLEDGEMEnts}

Part of this work was carried out during the visit of the first author to the University of São Paulo in 2009. This author would like to thank the University of São Paulo for their hospitality and the excellent working conditions, and Fapesp (2008/10471-2) for financial support.

\section{REFERENCES}

[1] Stephen Berman and Yuly Billig, Irreducible representations for toroidal Lie algebras, J. Algebra 221 (1999), no. 1, 188-231, DOI 10.1006/jabr.1999.7961. MR.1722910(2000k:17004)

[2] Yuly Billig, A category of modules for the full toroidal Lie algebra, Int. Math. Res. Not., posted on 2006, Art. ID 68395, 46, DOI 10.1155/IMRN/2006/68395. MR2272091 (2007h:17030)

[3] Yuly Billig, Alexander Molev, and Ruibin Zhang, Differential equations in vertex algebras and simple modules for the Lie algebra of vector fields on a torus, Adv. Math. 218 (2008), no. 6, 1972-2004, DOI 10.1016/j.aim.2008.03.026. MR.2431666 (2009e:17042)

[4] Yuly Billig and Kaiming Zhao, Weight modules over exp-polynomial Lie algebras, J. Pure Appl. Algebra 191 (2004), no. 1-2, 23-42, DOI 10.1016/j.jpaa.2003.12.004. MR2048305 (2005a:17021)

[5] Chongying Dong, Haisheng Li, and Geoffrey Mason, Vertex Lie algebras, vertex Poisson algebras and vertex algebras, Recent developments in infinite-dimensional Lie algebras and conformal field theory (Charlottesville, VA, 2000), Contemp. Math., vol. 297, Amer. Math. Soc., Providence, RI, 2002, pp. 69-96, DOI 10.1090/conm/297/05093. MR1919813 (2003h:17035)

[6] S. Eswara Rao, Irreducible representations of the Lie-algebra of the diffeomorphisms of a d-dimensional torus, J. Algebra 182 (1996), no. 2, 401-421, DOI 10.1006/jabr.1996.0177. MR 1391590 (97f:17007)

[7] S. Eswara Rao, Partial classification of modules for Lie algebra of diffeomorphisms of $d$ dimensional torus, J. Math. Phys. 45 (2004), no. 8, 3322-3333, DOI 10.1063/1.1769104. MR2077512(2005d:17028)

[8] Edward Frenkel, Victor Kac, Andrey Radul, and Weiqiang Wang, $\mathcal{W}_{1+\infty}$ and $\mathcal{W}\left(\mathfrak{g l}_{N}\right)$ with central charge N, Comm. Math. Phys. 170 (1995), no. 2, 337-357. MR1334399 (96i:17024)

[9] I. B. Frenkel, Representations of affine Lie algebras, Hecke modular forms and Korteweg-de Vries type equations, Lie algebras and related topics (New Brunswick, N.J., 1981), Lecture Notes in Math., vol. 933, Springer, Berlin-New York, 1982, pp. 71-110. MR675108(84f:17006)

[10] Igor B. Frenkel, Yi-Zhi Huang, and James Lepowsky, On axiomatic approaches to vertex operator algebras and modules, Mem. Amer. Math. Soc. 104 (1993), no. 494, viii+64, DOI 10.1090/memo/0494. MR.1142494 (94a:17007)

[11] V. M. Futorny, Irreducible non-dense $A_{1}^{(1)}$-modules, Pacific J. Math. 172 (1996), no. 1, 83-99. MR.1379287 (96m:17042)

[12] Victor Kac, Vertex algebras for beginners, 2nd ed., University Lecture Series, vol. 10, American Mathematical Society, Providence, RI, 1998. MR1651389 (99f:17033)

[13] V. G. Kac, D. A. Kazhdan, J. Lepowsky, and R. L. Wilson, Realization of the basic representations of the Euclidean Lie algebras, Adv. in Math. 42 (1981), no. 1, 83-112, DOI 10.1016/0001-8708(81)90053-0. MR633784(83a:17016)

[14] Christian Kassel, Kähler differentials and coverings of complex simple Lie algebras extended over a commutative algebra, Proceedings of the Luminy conference on algebraic $K$-theory (Luminy, 1983), 1984, pp. 265-275, DOI 10.1016/0022-4049(84)90040-9. MR772062 (86h:17013)

[15] Fons ten Kroode and Johan van de Leur, Bosonic and fermionic realizations of the affine algebra $\widehat{\mathrm{gl}}_{n}$, Comm. Math. Phys. 137 (1991), no. 1, 67-107. MR.1099256 (92f:17030) 
[16] James Lepowsky and Robert Lee Wilson, Construction of the affine Lie algebra $A_{1}(1)$, Comm. Math. Phys. 62 (1978), no. 1, 43-53. MR0573075 (58 \#28089)

[17] Hai-Sheng Li, Local systems of vertex operators, vertex superalgebras and modules, J. Pure Appl. Algebra 109 (1996), no. 2, 143-195, DOI 10.1016/0022-4049(95)00079-8. MR1387738 (97d:17016)

[18] Fyodor Malikov and Vadim Schechtman, Chiral de Rham complex. II, Differential topology, infinite-dimensional Lie algebras, and applications, Amer. Math. Soc. Transl. Ser. 2, vol. 194, Amer. Math. Soc., Providence, RI, 1999, pp. 149-188. MR1729362 (2000j:17035b)

[19] Fyodor Malikov and Vadim Schechtman, Chiral Poincaré duality, Math. Res. Lett. 6 (1999), no. 5-6, 533-546, DOI 10.4310/MRL.1999.v6.n5.a6. MR1739212 (2000m:14019)

[20] Fyodor Malikov, Vadim Schechtman, and Arkady Vaintrob, Chiral de Rham complex, Comm. Math. Phys. 204 (1999), no. 2, 439-473, DOI 10.1007/s002200050653. MR.1704283 (2000j:17035a)

[21] Olivier Mathieu, Classification of Harish-Chandra modules over the Virasoro Lie algebra, Invent. Math. 107 (1992), no. 2, 225-234, DOI 10.1007/BF01231888. MR1144422(93d:17034)

[22] Olivier Mathieu, Classification of irreducible weight modules (English, with English and French summaries), Ann. Inst. Fourier (Grenoble) 50 (2000), no. 2, 537-592. MR.1775361 (2001h:17017)

[23] Volodymyr Mazorchuk and Kaiming Zhao, Supports of weight modules over Witt algebras, Proc. Roy. Soc. Edinburgh Sect. A 141 (2011), no. 1, 155-170, DOI 10.1017/S0308210509000912. MR2773444(2012d:17021)

[24] Robert V. Moody and Arturo Pianzola, Lie algebras with triangular decompositions, Canadian Mathematical Society Series of Monographs and Advanced Texts, John Wiley \& Sons, Inc., New York, 1995. A Wiley-Interscience Publication. MR1323858 (96d:17025)

[25] Mirko Primc, Vertex algebras generated by Lie algebras, J. Pure Appl. Algebra 135 (1999), no. 3, 253-293, DOI 10.1016/S0022-4049(97)00144-8. MR1670692(2000c:17046)

[26] Michael Roitman, On free conformal and vertex algebras, J. Algebra 217 (1999), no. 2, 496527, DOI 10.1006/jabr.1998.7834. MR 1700512(2001i:17036)

[27] A. N. Rudakov, Irreducible representations of infinite-dimensional Lie algebras of Cartan type (Russian), Izv. Akad. Nauk SSSR Ser. Mat. 38 (1974), 835-866. MR0360732 (50 \#13179)

School of Mathematics and Statistics, Carleton University, Ottawa, Ontario, Canada K1S 5B6

E-mail address: billig@math.carleton.ca

Instituto de Matemática e Estatística, Universidade de São Paulo, São Paulo, 05315970 BRASIL

E-mail address: futorny@ime.usp.br 